\title{
Signatures from a merging galaxy cluster and its AGN population: LOFAR observations of Abell 1682
}

\author{
A. O. Clarke ${ }^{1}$, A. M. M. Scaife ${ }^{1}$, T. Shimwell ${ }^{3,2}$, R. J. van Weeren ${ }^{2}$, A. Bonafede ${ }^{4,5}$, G. Heald ${ }^{6,3,7}$, G. Brunetti ${ }^{4,5}$, \\ T. M. Cantwell ${ }^{1}$, F. de Gasperin ${ }^{8}$, M. Brüggen ${ }^{8}$, A. Botteon ${ }^{4,5}$, M. Hoeft ${ }^{11}$, C. Horellou ${ }^{9}$, R. Cassano ${ }^{4,5}$, \\ J. J. Harwood ${ }^{10}$, and H. J. A. Röttgering ${ }^{2}$
}

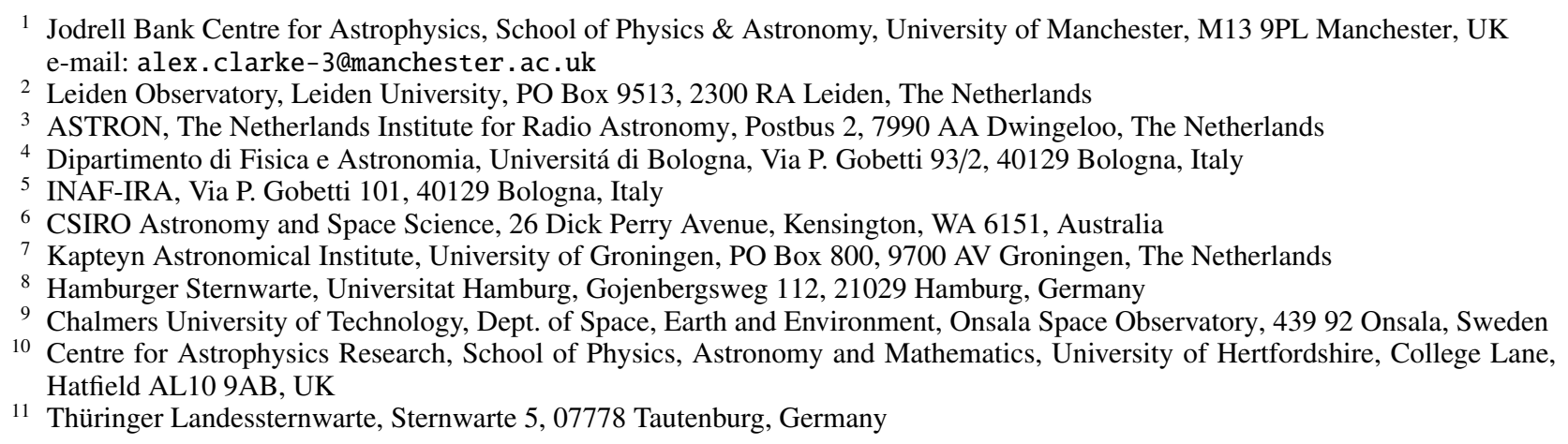

Received 29 March 2019 / Accepted 12 June 2019

\begin{abstract}
We present LOFAR data from 110-180 MHz of the merging galaxy cluster Abell 1682, alongside archival optical, radio, and X-ray data. Our images of 6 arcsec in resolution at low frequencies reveal new structures associated with numerous radio galaxies in the cluster. At a resolution of 20 arcsec we see diffuse emission throughout the cluster over hundreds of kiloparsecs, indicating particle acceleration mechanisms are in play as a result of the cluster merger event and powerful active galactic nuclei. We show that a significant part of the cluster emission is from an old radio galaxy with very steep spectrum emission (having a spectral index of $\alpha<-2.5)$. Furthermore, we identify a new region of diffuse steep-spectrum emission $(\alpha<-1.1)$ as a candidate for a radio halo which is co-spatial with the centre of the cluster merger. We suggest its origin as a population of old and mildly relativistic electrons left over from radio galaxies throughout the cluster which have been re-accelerated to higher energies by shocks and turbulence induced by the cluster merger event. We also note the discovery of six new giant radio galaxies in the vicinity of Abell 1682.
\end{abstract}

Key words. galaxies: clusters: intracluster medium - galaxies: halos - radiation mechanisms: non-thermal - galaxies: jets radio continuum: galaxies - quasars: supermassive black holes

\section{Introduction}

Clusters of galaxies are the largest gravitationally bound structures in the Universe and arise primarily at filament junctions in the cosmic web. Under the influence of strong gravitational fields at these junctions, it becomes increasingly likely that clusters will collide or merge, which can release $10^{64} \mathrm{erg}$ of gravitational potential energy into the intra-cluster medium (ICM). This energy heats up the gas interwoven between the hundreds of galaxies and produces shocks and turbulence throughout the ICM (Brunetti \& Jones 2014).

Owing to the presence of large-scale micro-Gauss-level magnetic fields throughout the cluster, the electrons in the ICM can produce synchrotron radiation at radio wavelengths (see van Weeren et al. 2019 for a recent review). Different acceleration mechanisms produce quite different observational features, namely polarised elongated structures called relics, and diffuse low-surface-brightness unpolarised structures called halos. A combination of diffusive shock acceleration (Blandford \& Eichler 1987) and shock drift acceleration (Ensslin et al. 1998;
Guo et al. 2014a,b) can explain the observed energetics of many relics, but other examples (van Weeren et al. 2016a; Botteon et al. 2016; Eckert et al. 2016) require additional constraints where the seed electrons are already mildly relativistic instead of being accelerated from the thermal pool. A prominent example of shock acceleration is shown by van Weeren et al. (2017) who present a relic being connected and illuminated by the jet of a radio galaxy.

In the favoured model for radio halo formation, a seed population of old relativistic electrons or secondary particles is re-accelerated to ultra-relativistic energies $(\mathrm{GeV})$ by turbulence induced by a cluster merger event (e.g. Brunetti et al. 2001; Petrosian 2001; Brunetti \& Lazarian 2007, 2011; Brunetti \& Jones 2014; Pinzke et al. 2015, 2017). This process also gives rise to a relatively new and elusive population of ultra-steepspectrum radio halos (USSRH; Brunetti et al. 2008; Cassano et al. 2006) that show up at very low frequencies. Furthermore, throughout galaxy clusters there are often many radio galaxies, which can produce very bright synchrotron emission from relativistic jets that extend throughout the cluster. These radio galax- 

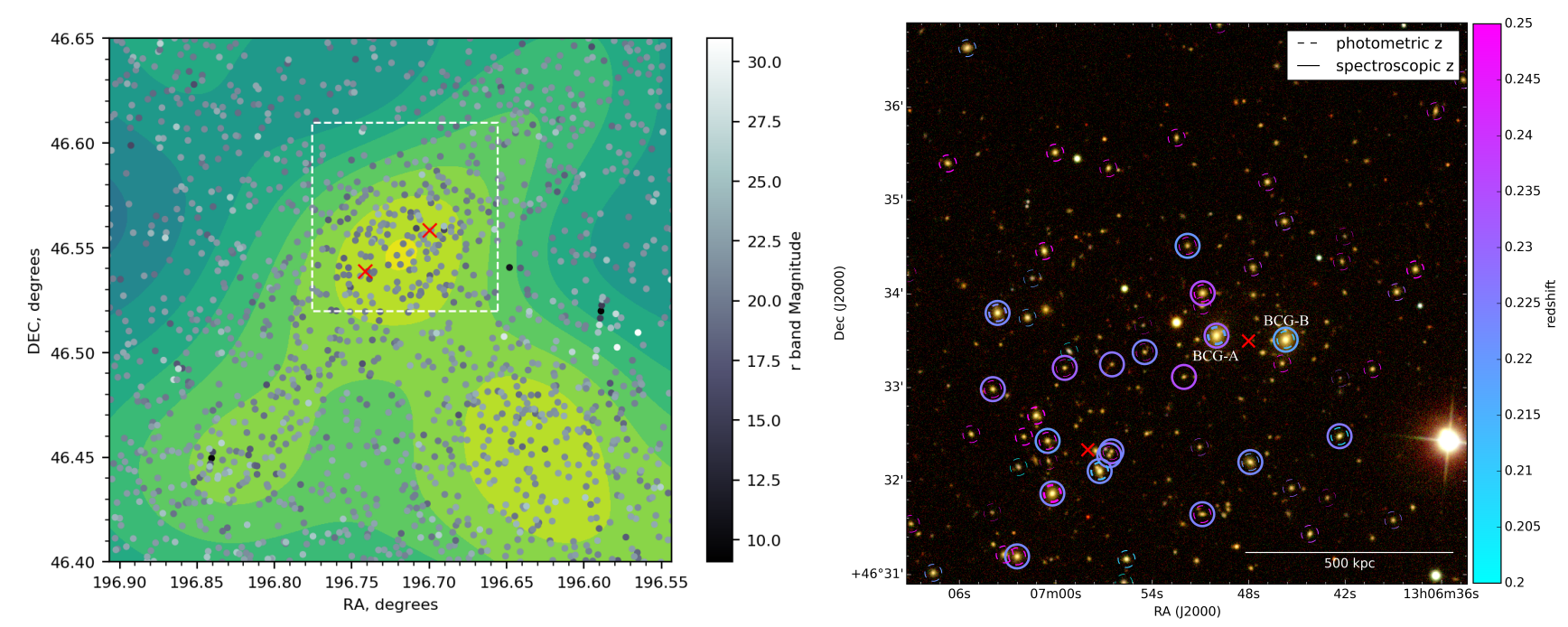

Fig. 1. Left: dots showing all galaxies identified in the region from SDSS, with the corresponding galaxy density shown by filled contours. Red crosses denote the centre of mass of the sub-clusters identified by Dahle et al. (2002). Right: SDSS composite image from bands $g, r, i$ within the area shown by the white square in the left plot. The two brightest galaxies are labelled, where BCG-A (13:06:49.99, +46:33:33.35) has a redshift of 0.232, and and BCG-B (13:06:45.69, +46:33:30.74) has a redshift of 0.218. The width of the dashed circles around galaxies with photometric redshifts is proportional to the error on the measurement, where they start at $4 \%$ and get thinner as the error increases. The maximum photometric error is $50 \%$.

ies are candidates for providing a seed population of mildly relativistic electrons, and there is evidence that shocks from clustermerger events have interacted with the tails of old radio galaxies (Enßlin \& Gopal-Krishna 2001; van Weeren et al. 2017; de Gasperin et al. 2017).

The LOw Frequency ARray (LOFAR; van Haarlem et al. 2013 ) is a telescope based in the Netherlands that is an effective instrument for observing synchrotron radiation in galaxy clusters, as shown by recent observations from van Weeren et al. (2016a), Shimwell et al. (2016), Hoang et al. (2017), Wilber et al. (2018), and Botteon et al. (2018). The results of this paper are based on observations performed with the LOFAR high-band antennas (HBA) at $110-180 \mathrm{MHz}$. With an excellent $u v$ coverage, and operation at these low frequencies, LOFAR has the novel capability of detecting and resolving the synchrotron emission that we expect to see in the ICM of merging clusters at arcsecond resolution.

This paper is organised as follows. In Sect. 2 we introduce Abell 1682 and summarise previous studies. In Sect. 3 we present new LOFAR observations, archival Giant Meterwavelength Radio Telescope (GMRT; Swarup et al. 1991) observations, and an analysis of the spectral index. In Sect. 4 we analyse and discuss the observational data, and we draw conclusions in Sect. 5. Throughout this paper we use the J2000 coordinate system, and adopt the spectral index convention that $S \propto v^{\alpha}$, where $S$ is the flux density, $v$ is the frequency, and $\alpha$ is the spectral index. We assume a flat Universe with $\Omega_{\mathrm{m}}=0.286, \Omega_{\Lambda}=0.714$, and $\mathrm{H}_{0}=67.8 \mathrm{~km} \mathrm{~s}^{-1} \mathrm{Mpc}^{-1}$ (Planck Collaboration XIII 2016), unless explicitly stated otherwise.

\section{Abell 1682}

A1682 is a massive merging galaxy cluster, with an X-ray luminosity $L_{\mathrm{X}}[0.1-2.4 \mathrm{keV}]=11.26 \times 10^{44} \mathrm{erg} \mathrm{s}^{-1}$ (assuming $\mathrm{H}_{0}=$ $50 \mathrm{~km} \mathrm{~s}^{-1} \mathrm{Mpc}^{-1}$; Ebeling et al. 1998). It has a total mass of the order of $10^{15} M_{\odot}$ and is shown to have a bi-modal mass distribution indicating a pre- or post-merger system (Dahle et al. 2002). The mass inferred from the Sunyaev-Zeldovich effect is $M_{500}=6.20_{-0.46}^{+0.45} \times 10^{14} M_{\odot}$ and $M_{500}=5.70 \pm 0.35 \times$
$10^{14} M_{\odot}$ after correction for diffuse Galactic plane emission (Planck Collaboration XXXII 2015; Planck Collaboration XXVII 2016). The left panel of Fig. 1 shows surrounding galaxies identified in the Sloan Digital Sky Survey (SDSS; Eisenstein et al. 2011) Data Release 13 (DR13; Albareti et al. 2017) along with a density plot. This agrees with Dahle et al. (2002) and shows a significant galaxy density enhancement approximately $1 \mathrm{Mpc}$ to the southeast of A1682.

The cluster has two dominant elliptical brightest cluster galaxies (BCGs) marked in the right panel of Fig. 1. The galaxy BCG-A (13:06:49.99, +46:33:33.35) is at a redshift of 0.232, has a velocity dispersion of $298 \pm 9 \mathrm{~km} \mathrm{~s}^{-1}$, and an estimated mass of $\log \left(M_{\mathrm{gal}} / M_{\odot}\right)=11.93$. The other, BCG-B (13:06:45.69, $+46: 33: 30.74)$, is at a redshift of 0.218 , has a velocity dispersion of $332 \pm 13 \mathrm{~km} \mathrm{~s}^{-1}$, and an estimated mass of $\log \left(M_{\mathrm{gal}} / M_{\odot}\right)=$ 11.81. These spectroscopic redshifts and masses are taken from SDSS DR13 (Albareti et al. 2017), where the redshift errors are $1 \times 10^{-5}$. Figure 2 shows a histogram of all spectroscopically and photometrically identified galaxies within a $6^{\prime}$ radius of the cluster centre. We take the redshift of this cluster as the average of the two BCGs, that is $z=0.225$ (Fig. 2), which matches well with the peak of the redshift histogram in Fig. 2. Older spectroscopic measurements of these galaxies show a very similar average cluster redshift of $z=0.226$ (Allen et al. 1992).

Galaxies identified with photometric redshifts have a mean error of $18 \%$, where the lowest is $4 \%$ and the highest $50 \%$ (Eisenstein et al. 2011). Despite the 4\% error on the photometric redshift for BCG-A, it is significantly different to the spectroscopic redshift (see the right panel of Fig. 1). Furthermore, as shown in Fig. 2, overall the photometric redshift measurements are biased towards higher redshifts. Many of them have significant flags in the training set coverage and fitted parameters (Beck et al. 2016), making their error estimate unreliable. Despite these caveats, they do still allow a basic identification of the brightest cluster members in the region. Given these problems with the photometric redshifts of cluster members, in figures throughout this paper we only highlight cluster member galaxies with photometric redshifts in the range $0.2-0.25$. However, many of the galaxies in the vicinity of Abell 1682 are resolved, appearing 


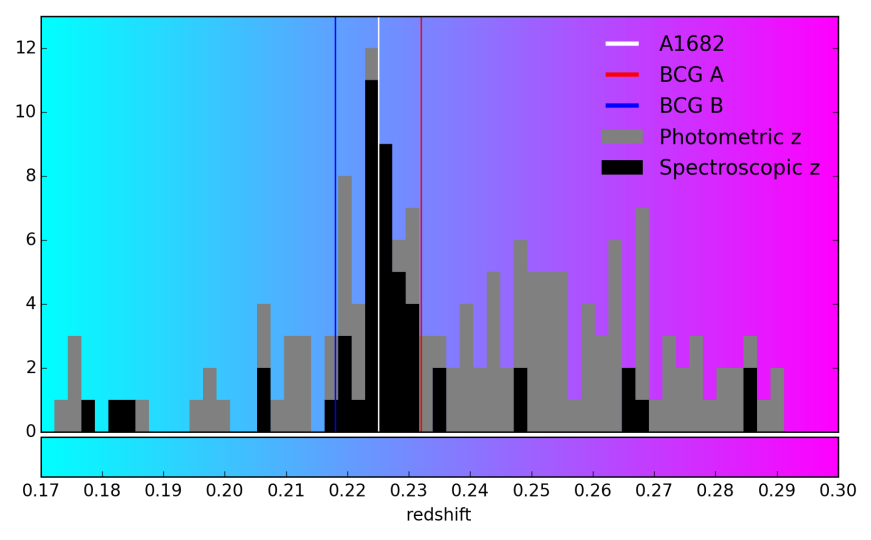

Fig. 2. Stacked histogram of galaxies with photometric and spectroscopic redshifts within a $6^{\prime}$ radius around A1682. The redshift of A1682 is calculated as the average of BCG-A and BCG-B, which aligns well with the peak of the histogram.

as likely cluster members even with photometric redshifts much larger than the average cluster redshift (up to $z=0.3$, as seen in Fig. 2).

Previous radio studies (Venturi et al. 2008, 2011, 2013; Macario et al. 2013) show that A1682 hosts complex clusterwide radio emission without a consensus on its origin. Venturi et al. (2008) present X-ray data, indicating that there are two subclusters which have passed through each other in a northwest to southeast direction (a post-merger scenario). The merger axis appears to be in the plane of the sky, however there is no information available on what the $3 \mathrm{D}$ velocity and momentum distribution is between the two clusters. Venturi et al. (2008) also show the first deep high-resolution radio observations at $610 \mathrm{MHz}$ using the GMRT, revealing extended emission (100-500 kpc) resembling radio jets or radio relics, but without a confident assessment of their origin.

Follow-up observations at $240 \mathrm{MHz}$ with the GMRT (Venturi et al. 2013) show structures very similar to those seen at $610 \mathrm{MHz}$. They also show higher-resolution (1.1") VLA data at $1.4 \mathrm{GHz}$ of BCG-B showing clear evidence of radio jets (less than $50 \mathrm{pc}$ in size) emanating from an AGN in the centre of this galaxy (see their Fig. 7). In their VLA data the radio lobes are brightest on the northeast side, becoming fainter towards the southwest, potentially suggesting that this radio galaxy (BCG-B) is moving northeast. However, the radio lobes could simply be expanding into a less-dense region of the cluster towards the southwest whilst the host galaxy does not move. Although radio jets can significantly change direction in a dense cluster environment, it is conceivable that the two $500 \mathrm{kpc}$ radio features connected to BCG-B are jets and lobes originating from this AGN. However, their spectral index map from $240 \mathrm{MHz}$ to $610 \mathrm{MHz}$ conflicts with this argument, as the NW ridge shows a spectral index gradient perpendicular to the major axis of the structure from $\alpha \approx-1$ to $\alpha \approx-2.3$. This is indicative of a radio relic, where the spectrum is flatter at the shock front $(\alpha \approx-0.6)$ and then steepens $(\alpha<-1)$ in its wake (e.g. Stroe et al. 2014). The orientation of this structure is perpendicular to the merger axis identified by the X-ray and galaxy distribution data, however a complex merger scenario and line-of-sight projection affects do not rule out the possibility that this is a relic.

The data at $240 \mathrm{MHz}$ also reveal new emission not seen at higher frequencies, with the detection of an area of diffuse radio emission, which becomes significant at lower resolution. This component is also seen in the Very Large Array Sky Survey (VLSSr; Lane et al. 2014) at $74 \mathrm{MHz}$, where Venturi et al.
(2013) calculate a spectral index between these two frequencies integrating over all the observed emission, with a value of $\alpha=-2.09 \pm 0.15$. However, we caution against using this value due to the different spatial scales that the GMRT and VLA probe given their configurations. Venturi et al. (2011) attempted to subtract the high-resolution data from both the 240 and $610 \mathrm{MHz}$ data, showing significant residuals over the whole cluster, particularly coincident with the diffuse component. The most significant detection of the diffuse component is from observations at $150 \mathrm{MHz}$ with the GMRT (Macario et al. 2013). Despite the low sensitivity of this observation (with an rms noise of $1.7 \mathrm{mJy}$ beam $^{-1}$ ), the diffuse component is clearly detected, with an integrated flux of $98 \pm 20 \mathrm{mJy}$. Macario et al. (2013) present a spectral index integrated over the whole emission from $150 \mathrm{MHz}$ to $240 \mathrm{MHz}$ of $\alpha=-1.7 \pm 0.1$, which is flatter than the value calculated from 240 to $610 \mathrm{MHz}$, and they suggest that this is due to the lower-surface-brightness sensitivity of observations at $150 \mathrm{MHz}$.

\section{Data}

\subsection{LOFAR calibration and imaging}

The LOFAR HBA observation presented here used 23 of the core Dutch stations (each comprising two sub-stations) and 13 remote Dutch stations. The central frequency was $150.0 \mathrm{MHz}$, with a total bandwidth of $70.3 \mathrm{MHz}$ from $111.5 \mathrm{MHz}$ to $181.8 \mathrm{MHz}$. The primary flux calibrator (3C295) was observed for 5 minutes and the target $(13: 06: 49.70+46: 32: 59.00)$ was observed with a total integration time of 9.67 hours. The separation between the calibrator and target sources is 11.9 degrees. The observation started on 19 April 2013 at 19:06:02.50, and finished on 20 April 2013 at 04:46:00.80 (UTC), however all data after 03:00:00 (CET) were flagged due to very poor ionospheric conditions. In total we used 8 hours of data in the calibration and imaging. We used the flux scale defined by Scaife \& Heald (2012) for 3C295, where the error on the flux scale for 3C295 is $4 \%$ for LOFAR HBA frequencies. However, as discussed in Shimwell et al. (2019) we take a conservative estimate of the flux-scale uncertainty as $20 \%$ given inadequate knowledge of the LOFAR beam during calibration, and note that sources crossmatched with the Tata GMRT Sky Survey (TGSS-ADR1; Intema et al. 2017) are consistent within this error.

We follow the calibration and imaging scheme described in detail in Shimwell et al. (2019) which accounts for directiondependent effects and wide field spectral deconvolution. In summary, this begins with a direction-independent calibration (described in detail by van Weeren et al. 2016b and Williams et al. 2016) and makes use of the LOFAR Default Preprocessing Pipeline (DPPP; van Diepen \& Dijkema 2018) for averaging and calibration and BlackBoard Selfcal (BBS; Pandey et al. 2009) for calibration. For the direction-dependent calibration, KillMS (kMS; Tasse 2014; Smirnov \& Tasse 2015) is used to calculate the Jones matrices and DDFacet (Tasse et al. 2018) is used to apply these during the imaging. The main software packages and the pipeline are publicly available and documented $^{1}$.

Figure 4 shows the high-resolution LOFAR HBA image. This used a Briggs (Briggs 1995) robust parameter of -0.5 to achieve a resolution of $6.0^{\prime \prime} \times 6.0^{\prime \prime}$, with an $\mathrm{rms}$ noise of $90 \mu \mathrm{Jy} \mathrm{beam}^{-1}$, a $u v$-minimum of $0.1 k \lambda$ and no $u v$-maximum

\footnotetext{
1 See https://github.com/saopicc for kMS and DDFacet, and https://github.com/mhardcastle/ddf-pipeline for the associated LoTSS-DR1 pipeline.
} 


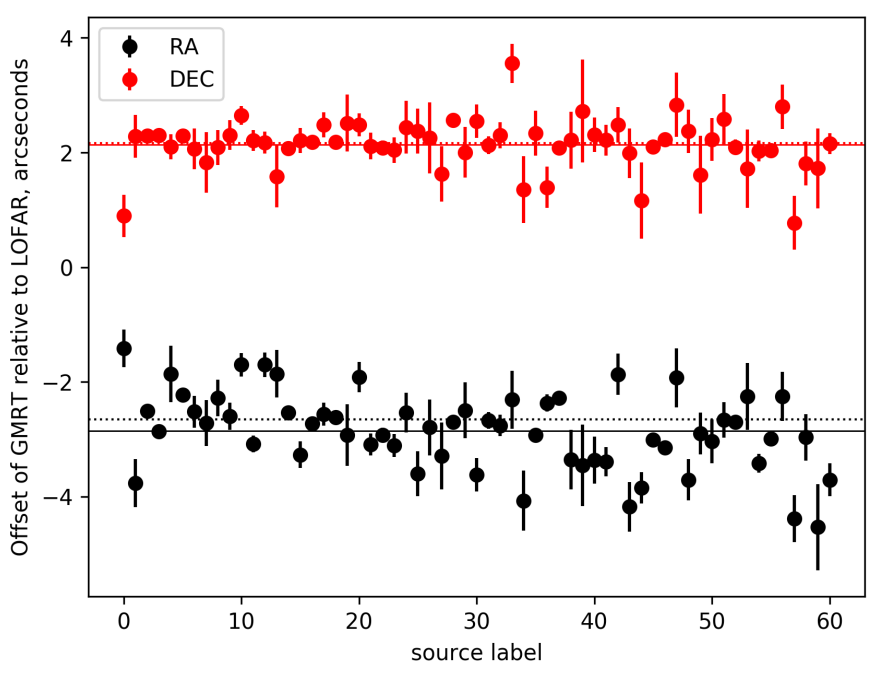

Fig. 3. Offset in RA and Dec of 61 sources crossmatched between the GMRT and LOFAR. The dashed line is the mean offset calculated from the original 92 sources, and the solid lines show the mean offset calculated after disregarding all sources more than five times their position error away from this mean in either RA or Dec. The final offset in RA and Dec was calculated to be 2.86 and 2.14 arcsec, respectively, and this correction was applied to the GMRT image.

limit. There are no significant direction-dependent errors visible and the rms noise is uniform around the source, measured at $90 \mu \mathrm{Jy}_{\mathrm{beam}}^{-1}$. The brightest pixel in the image is $0.29 \mathrm{Jy}_{\text {beam }}^{-1}$ (the peak of the colour-scale). There are small spurious areas with negative wells at -5 times the rms noise around the brightest part of the cluster. Figure 12 also shows the LOFAR data as a semi-transparent layer on top of the optical background from SDSS.

Figure 5 shows the low-resolution image. This used a Briggs robust parameter of -0.25 and a $u v$-range of $0.1-25.75 \mathrm{k} \lambda$ to achieve a resolution of $20^{\prime \prime} \times 20^{\prime \prime}$. The rms noise is $130 \mu \mathrm{Jybeam}^{-1}$, and the brightest pixel has a value of $0.74 \mathrm{Jy}_{\text {beam }}{ }^{-1}$. As in the high-resolution image, there are small spurious areas with negative wells at -5 times the rms noise around the brightest part of the cluster. At this lower resolution, extended emission is recovered more readily, and the patchy areas of emission seen at high resolution are robustly detected as areas of low-surface-brightness, diffuse emission. This is most prominent in the region between the centres of mass of the two clusters, and in regions towards the extremities of the extended structures.

Overall, we observe all the features identified in previous studies, plus some additional features. The diffuse component is now resolved, and distinguished from diffuse emission in the region between the centre of mass of each of the sub-clusters. In Sect. 4 we discuss the origin of these components.

\subsection{Spectral index maps with the GMRT}

We re-analysed archival GMRT data at $610 \mathrm{MHz}$ using the Source Peeling and Atmospheric Modelling software (SPAM; Intema 2014). Both the GMRT and LOFAR data sets were imaged using a $u v$-minimum of $0.1 \mathrm{k} \lambda$ at the same resolution of $6^{\prime \prime} \times 6^{\prime \prime}$. As described in Shimwell et al. (2019) the astrometry of our LOFAR images has been matched to optical sources in PanSTARRS (Flewelling et al. 2016), however our GMRT image was mis-aligned, and so we applied an astrometry correction to our GMRT image to align it with our LOFAR image. To do this we first masked A1682 and used PyBDSF (Mohan \& Rafferty 2015 ) to detect sources above five times the rms noise in both the GMRT and LOFAR images. We then crossmatched all sources detected by the GMRT with LOFAR using a matching radius of 6 arcsec. From the 92 sources detected by both the GMRT and LOFAR around A1682, we calculated a mean offset in RA and Dec of -2.65 and 2.16 arcsec, respectively (dashed black and red lines in Fig. 3). We then disregarded all sources that were more than five times their position error away from this mean (in either RA or Dec), leaving the 61 sources shown in Fig. 3, and repeated the calculation of the mean offset in RA and Dec giving 2.86 and 2.14 arcsec, respectively (the solid black and red lines in Fig. 3). This procedure ensured we only used reliably matched sources to correct the astrometry, and the final correction was applied to the GMRT image.

We used the Broadband Radio Astronomy ToolS (BRATS; Harwood et al. 2013, 2015) to calculate the spectral index value for each pixel and corresponding error map, as shown in Fig. 7. Only data above five times the rms noise in both data sets were used. We note that applying the $u v$-minimum of $0.1 k \lambda$ during imaging was done to ensure both telescopes were sampling the same spatial scales, since LOFAR has significantly better $u v$ coverage below this value. However, when doing this we only noticed a change in the spectral index value of around $1 \%$, indicating that the spatial scales involved in this source were not large enough to greatly affect the spectral index calculations.

\section{Analysis}

Throughout the cluster we observe complex radio emission with LOFAR at high and low resolution. The total integrated flux density (all emission above five times the rms noise) of Abell 1682 at $150 \mathrm{MHz}$ is $3.53 \pm 0.71 \mathrm{Jy}$ in both the $6^{\prime \prime}$ and $20^{\prime \prime}$ images (Figs. 4 and 5). It is challenging to disentangle contributions from the ICM, the BCGs, or other AGNs throughout the cluster. Here we discuss potential origins of each component considering ancillary radio, X-ray, and optical data, referring closely to Figs. 4-9 and sources labelled within.

\subsection{AGNs, radio jets, and lobes}

Figure 4 presents our 6" resolution LOFAR data showing the detailed morphology of the radio structures in this galaxy cluster. In these new data, BCG-A shows an extension of approximately $30^{\prime \prime}$ to the southwest (towards the red cross marking the centres of mass of the sub-clusters) resembling a radio jet from its AGN that has not been seen in previous data. However, we cannot rule out that this extension is a result of a different source of radio emission in this complex area of the cluster (another AGN), or has contamination from a high-redshift background AGN. The galaxy BCG-A is coincident with the peak in the X-ray image (Fig. 8), and whilst it hosts a radio-loud AGN capable of producing large radio jets, it is currently not clearly connected to the surrounding extended radio structures. BCG-A has a peak flux density of $27 \pm 4 \mathrm{mJy}_{\text {beam }}{ }^{-1}$ at $150 \mathrm{MHz}$, and fitting a single component Gaussian (ignoring the extension to the southwest) gives an integrated flux density of $44 \pm 7 \mathrm{mJy}$ at $150 \mathrm{MHz}$.

A prominent optical source in this region is labelled as RG-A, which could be a tailed radio source associated with the emission highlighted by the dotted green line (resembling a wide-angle tailed radio galaxy moving southeast). The spectral index in the region around RG-A is $\alpha=-1.70 \pm 0.19$ (150$610 \mathrm{MHz}$, see Fig. 7). BCG-A has an average spectral index 

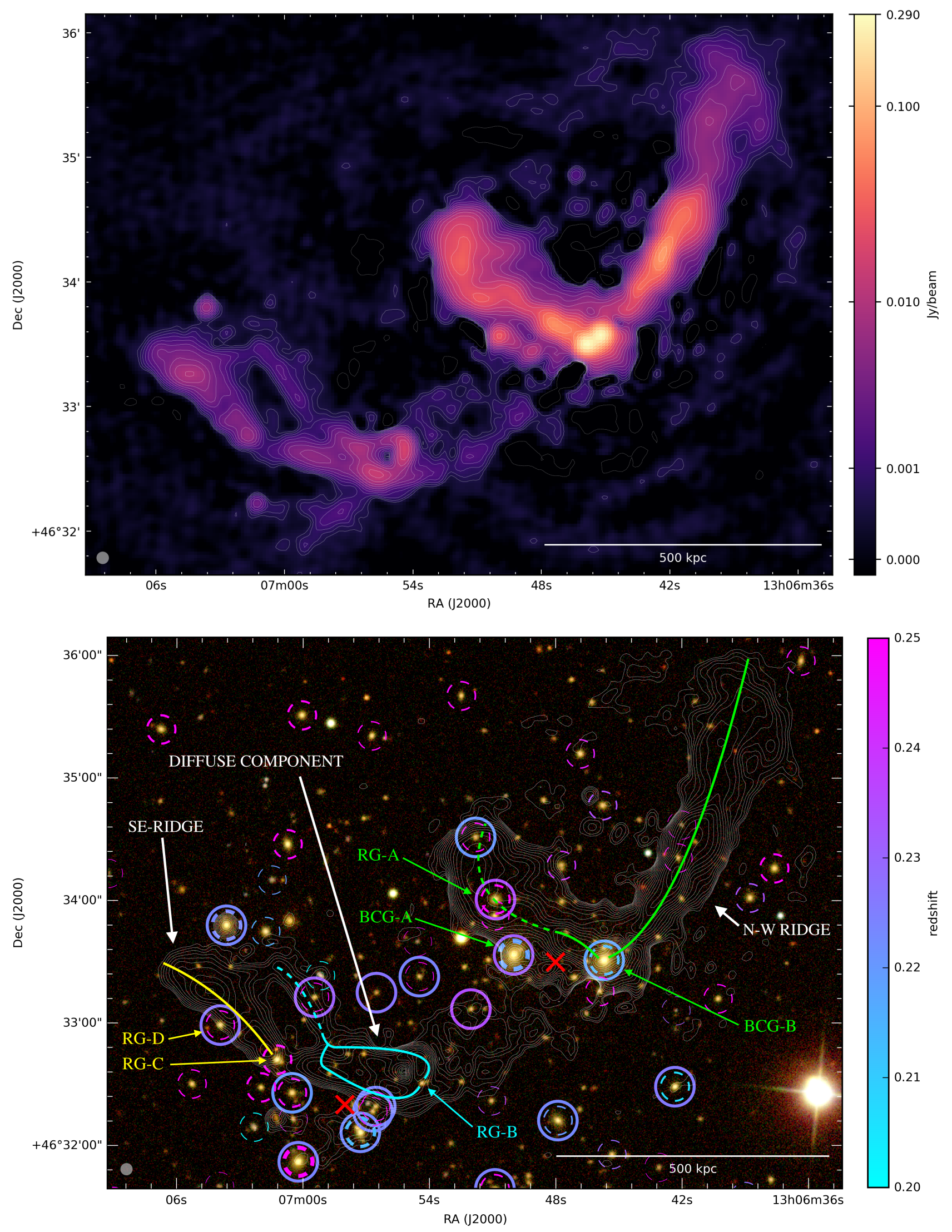

Fig. 4. Top: colour-scale image of A1682 on a log stretch from $-90 \mu \mathrm{Jy}_{\text {beam }}{ }^{-1}$ to $0.29 \mathrm{Jy} \mathrm{beam}^{-1}$ (the brightest pixel is $0.29 \mathrm{Jy}_{\text {beam }}{ }^{-1}$ ). The rms noise is $90 \mu \mathrm{Jy}_{\text {beam }}{ }^{-1}$. Dashed contours are overlaid at -5 times the rms noise. Solid contours start at 5 times the rms noise and increase by a factor of 1.4. The resolution is $6^{\prime \prime} \times 6^{\prime \prime}$. Bottom: SDSS background composite image from bands $g, r$, and $i$ with the same contours as above. Redshift coloured markers are the same as in Fig. 1. Radio-galaxy candidates (RG-A to RG-D) are marked, along with lines showing the possible orientation of their radio jets and/or tails. Red crosses mark the centres of mass of the two sub-clusters identified by Dahle et al. (2002). 


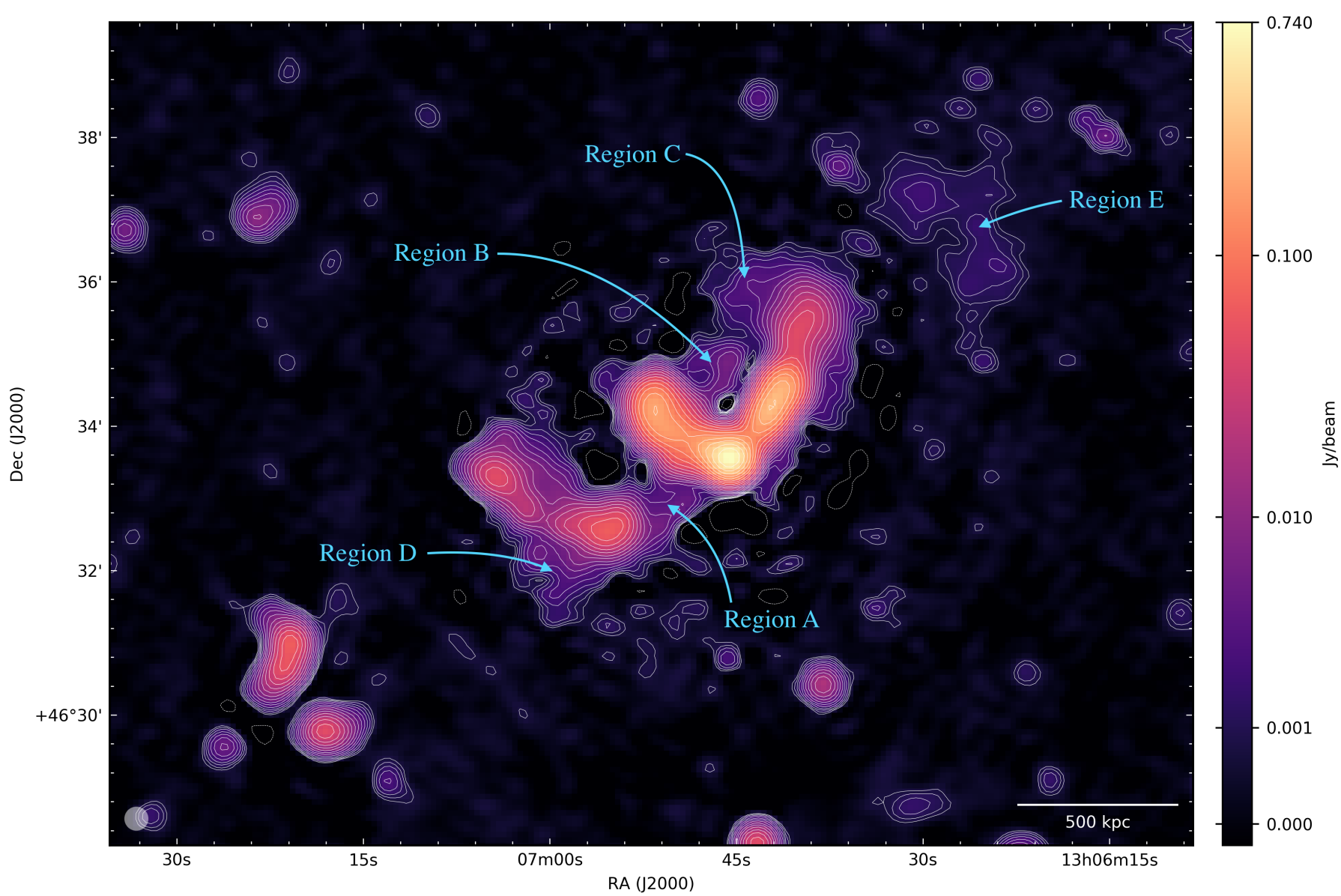

Fig. 5. Colour-scale image of A1682 on a $\log$ stretch from $-130 \mu \mathrm{Jy}_{\text {beam }}{ }^{-1}$ to $0.74 \mathrm{Jy} \mathrm{beam}^{-1}$ (the brightest pixel is $0.74 \mathrm{Jy}^{\mathrm{beam}}{ }^{-1}$ ). The rms noise is $130 \mu \mathrm{Jy}_{\text {beam }}{ }^{-1}$. Dashed contours are overlaid at -5 times the rms noise. Solid contours start at 5 times the rms noise and increase by a factor of 1.4. The resolution is $20^{\prime \prime} \times 20^{\prime \prime}$.

of $\alpha=-1.51 \pm 0.29$ calculated over the island of 74 pixels in Fig. 7. The lack of any flatter spectrum components aligned with RG-A or BCG-A suggest neither currently have active radio jets. If either BCG-A or RG-A are responsible for the extended radio emission in this area, regions $B$ and $C$ in Fig. 5 could be emission from earlier in their lifetime when they were positioned farther to the northwest. Alternatively, these regions could be a result of the radio lobes expanding out into less-dense regions of the ICM.

We note that to the southeast of RG-A the radio emission extends to overlap a large optical source; however this source is classified as a star in SDSS data. Our spectral index map indicates the emission flattens in this area, but the error is very large and we conclude it is unlikely there are any other optical counterparts responsible for this emission other than BCG-A, RG-A, or BCG-B.

BCG-B has a peak flux density of $0.29 \mathrm{Jy} \mathrm{beam}^{-1}$ in Fig. 4, and the component $1.2^{\prime \prime}$ to the northwest of this (likely to be where its jet has left the galaxy) has a peak flux density of $0.28 \mathrm{Jy} \mathrm{beam}^{-1}$. The average spectral index on top of BCG-B is $\alpha=-0.65 \pm 0.15$ (averaged over 56 pixels centred on BCG$\mathrm{B}$ which are above 1000 times the rms noise in the LOFAR image). There is a steady fall off in surface brightness going from BCG-B to the southwest, with little change in the perpendicular direction (northwest to southeast). This agrees with a scenario in which BCG-B is moving northeast with its radio jets oriented perpendicular to this direction of travel (as shown in Venturi et al. 2013). As such, at an earlier point in time the alignment of BCG-B could explain the resolved structures to the southwest of BCG-B in Fig. 4. However, some of this emission is seen spuriously in the GMRT image at $610 \mathrm{MHz}$ (Fig. 7) and is spatially coincident with a galaxy directly to the south of BCG-B with photometric redshift (indicating that it is a cluster member). The detection is not significant enough to obtain a reliable spectral index estimation. Optical sources surrounding BCG-B are also likely to be cluster members and could be contributing to the radio emission with AGNs of their own.

In the southeast of the cluster the emission consists of numerous AGNs identified as cluster members and two regions of steep-spectrum emission previously labelled as the SE ridge and the diffuse component. The SE ridge is resolved in these new data, and is likely associated with RG-C, -D, or -E in Fig. 4. Whilst RG-D is the only one with spectra in SDSS, RG-C and -E are very likely to be cluster members and have photometric redshifts of $0.254 \pm 0.021$ and $0.312 \pm 0.110$, respectively. The spectral index information presented in Figs. 7 and 9 indicates that the host is in the centre of this structure where the spectral index is flattest ( $\alpha=-0.83 \pm 0.12$ ). The spectrum of the emission then steepens either side of this (which is expected as the electrons age), reaching $\alpha=-1.64 \pm 0.15$ on the northeast side, and $\alpha=-1.11 \pm 0.26$ on the southwest side. The asymmetry of the spectral index profile around the central optical counterpart could be caused by differing density of the ICM, as seen in studies such as that by de Gasperin (2017). Given the spectral index 


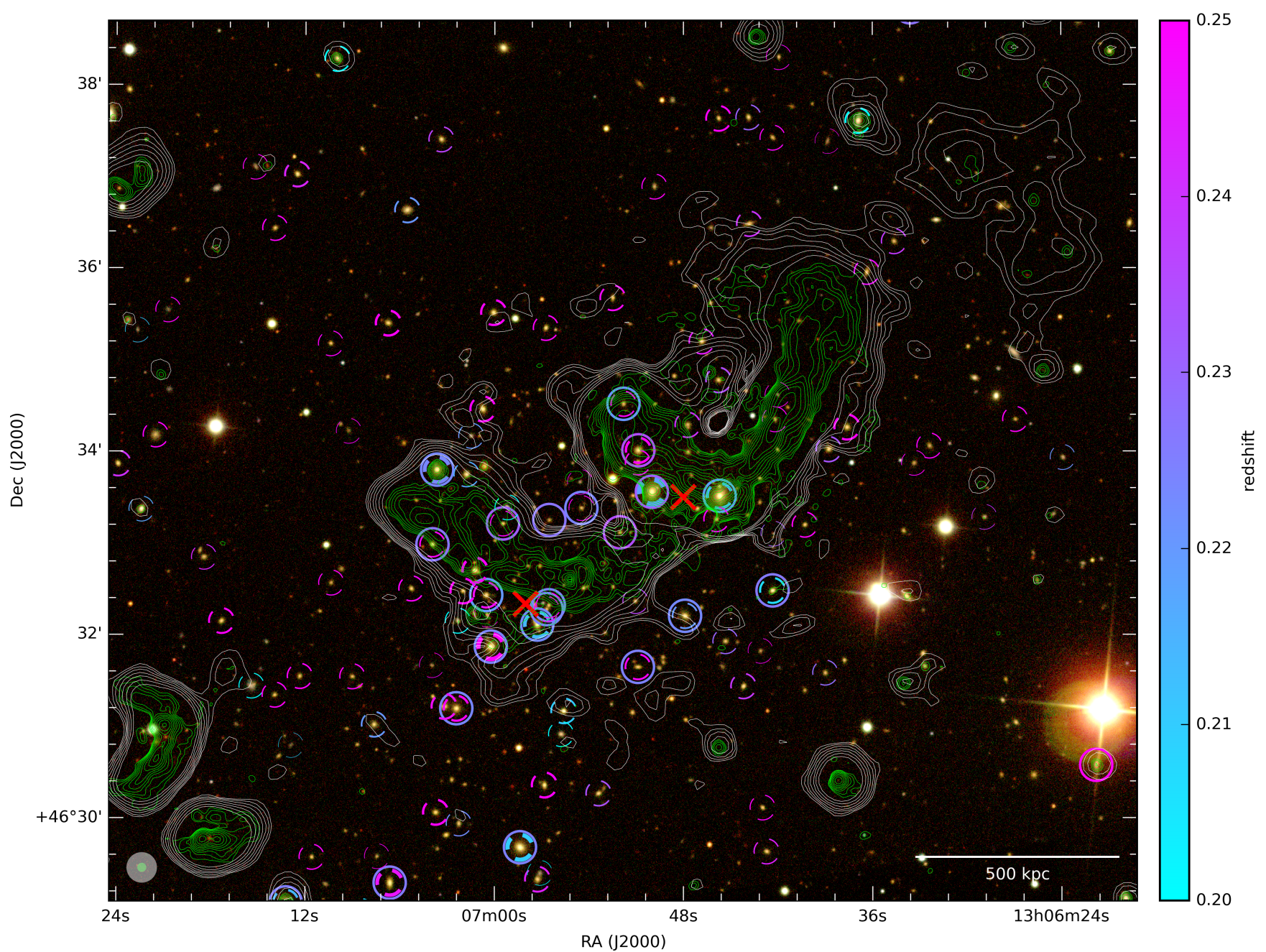

Fig. 6. SDSS background composite image from bands $g$, $r$, and $i$. LOFAR contours at $20^{\prime \prime}$ resolution are overlaid in white starting at five times the rms noise and increasing by a factor of 1.4. LOFAR contours at $6^{\prime \prime}$ resolution are overlaid in green starting at five times the rms noise and increasing by a factor of 1.6. Redshift coloured markers are the same as in Fig. 1. Red crosses mark the centres of mass of the two sub-clusters identified by Dahle et al. (2002).
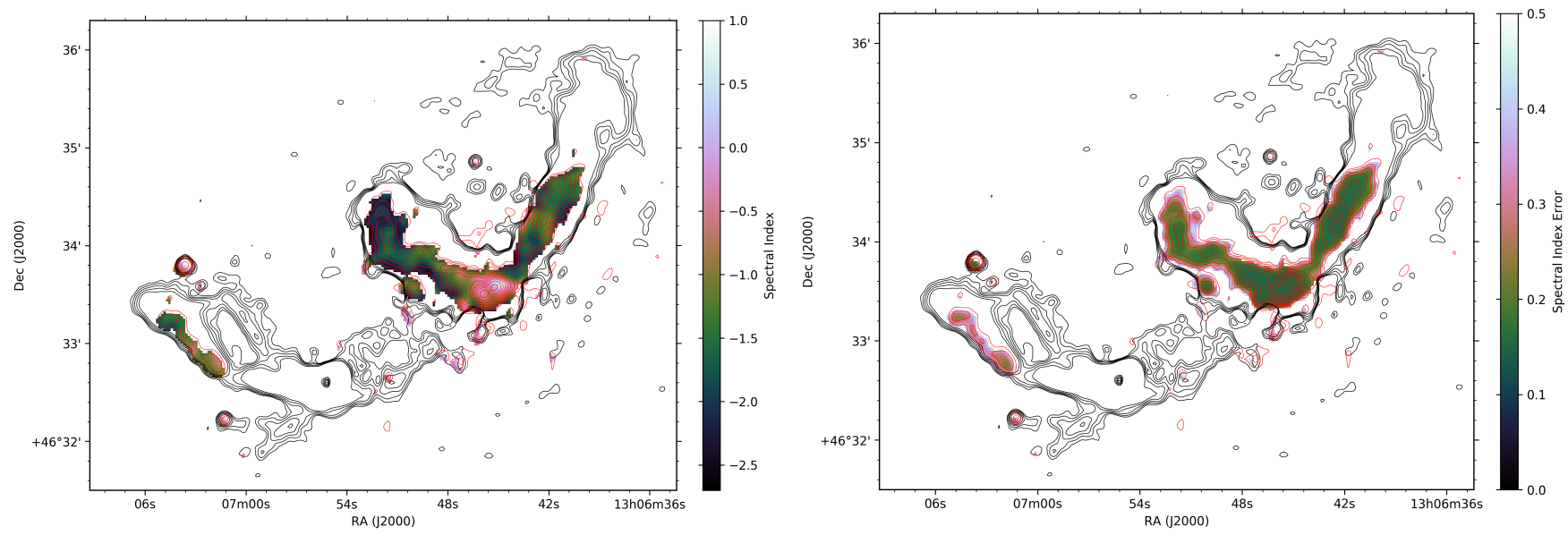

Fig. 7. Left: spectral index map from our reprocessed GMRT data at $610 \mathrm{MHz}$ to our LOFAR HBA data at $150 \mathrm{MHz}$. Both maps are at a resolution of $6^{\prime \prime} \times 6^{\prime \prime}$. Contours start at five times the rms noise $\left(90 \mu \mathrm{Jy} \mathrm{beam}^{-1}\right.$ for both LOFAR and GMRT), and increase by a factor of 1.7 . Right: corresponding spectral index error map for each pixel.

profile and morphology of the radio emission, the most likely optical counterpart is RG-D or -E in the centre where the spectrum is flattest, although RG-D is slightly to the southeast of the GMRT emission.
While RG-C appears to be a possible candidate in terms of its spatial location, the spectral index profile of the extended radio emission and lack of a flatter spectrum component near $\mathrm{RG}-\mathrm{C}$ suggests it is unlikely to be the cause. There is a drop in 


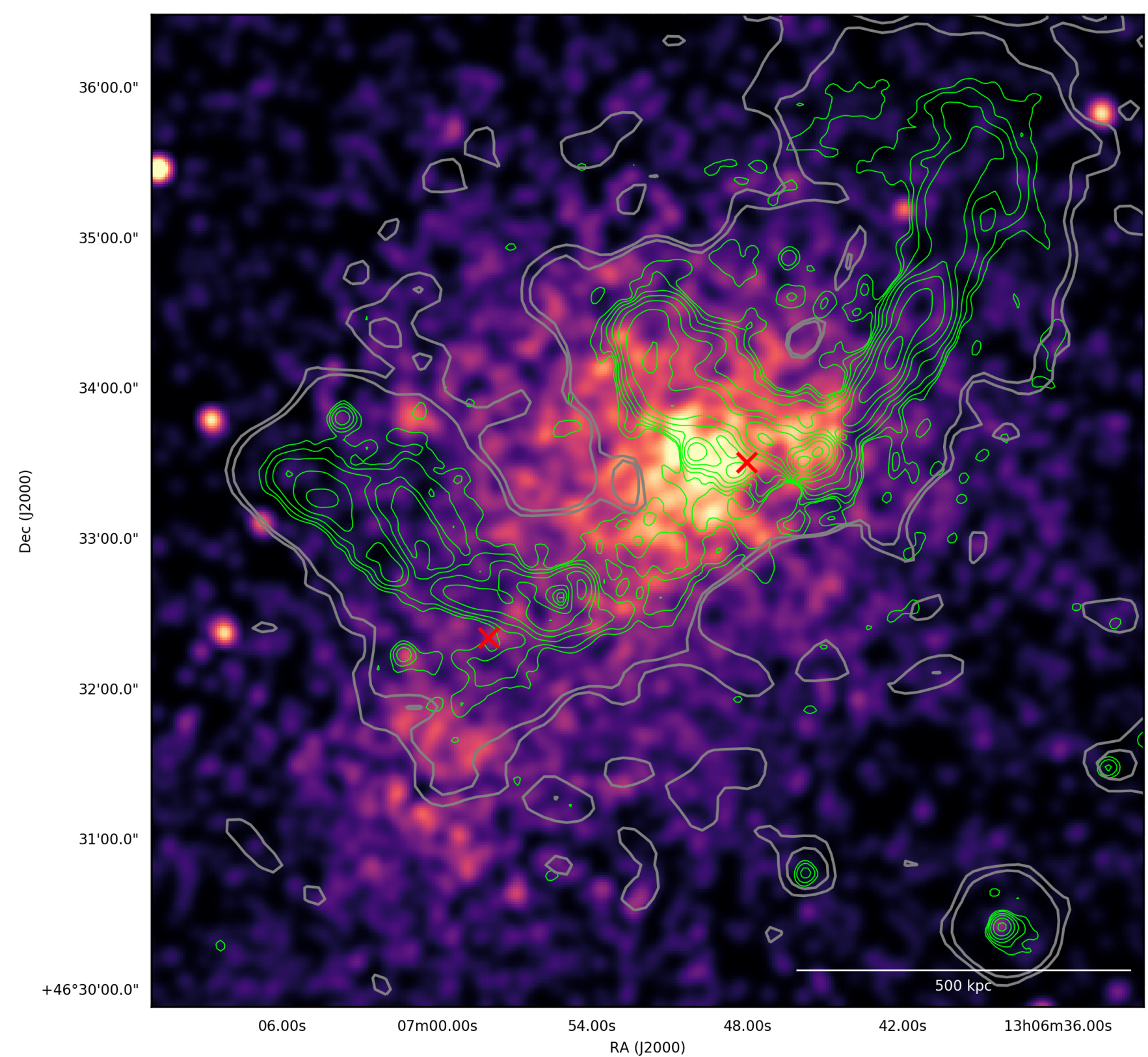

Fig. 8. X-ray mosaic from Chandra (ObsID's: 3244 and 11725) in the $0.5-2.0 \mathrm{keV}$ band, smoothed with a Gaussian kernel of standard deviation $5^{\prime \prime}$. LOFAR contours are overlaid in green starting at five times the rms noise $\left(90 \mu \mathrm{Jy}\right.$ beam $\left.^{-1}\right)$ and increase by factor of two, at a resolution of $6^{\prime \prime} \times 6^{\prime \prime}$. Grey contours shows LOFAR data at $20^{\prime \prime}$ resolution at five and ten times the rms noise $\left(130 \mu \mathrm{Jy} \mathrm{beam}^{-1}\right)$. Red crosses mark the centre of mass of each of the sub-clusters as in other figures.

the brightness of the LOFAR emission around RG-C as it connects to the diffuse component in the southwest. Furthermore, no GMRT emission (indicative of a counter-jet) is seen to the southwest of RG-C which would have been detected if it were of similar spectral index values. Therefore, emission to the southwest of RG-C appears to become significantly steeper. Whilst RG-C could potentially be the host of a large radio galaxy contributing to both the S-E ridge and diffuse component, overall we conclude that the S-E ridge is likely of a separate origin to the diffuse component that we discuss in Sect. 4.3.

\subsection{The NW ridge: a radio relic or lobe?}

The extended structure of the NW ridge resembles the jet and lobe of a radio galaxy originating from a powerful AGN in
BCG-B (green lines in Fig. 4). The orientation of the jets of BCG-B from the higher-resolution VLA observation (Venturi et al. 2013) agrees with this scenario. Furthermore, the AGN at the centre of BCG-B could be responsible for all the emission traced by green lines in Fig. 4. Our spectral index map from this LOFAR $150 \mathrm{MHz}$ observation to our reprocessed GMRT observation at $610 \mathrm{MHz}$ (Fig. 7) shows flat spectrum emission around BCG-B $(\alpha=-0.65 \pm 0.15)$, which becomes steep spectrum emission in both directions traced by green lines in Fig. $4(\alpha=-2.1 \pm 0.2$ in the east lobe and $\alpha=$ $-1.96 \pm 0.16$ in the NW ridge), which is typical of a large radio galaxy with lobes extending hundreds of kiloparsecs. However, there is also the possibility that the NW ridge is a radio relic, or has at least been partially re-accelerated by the cluster merger. 

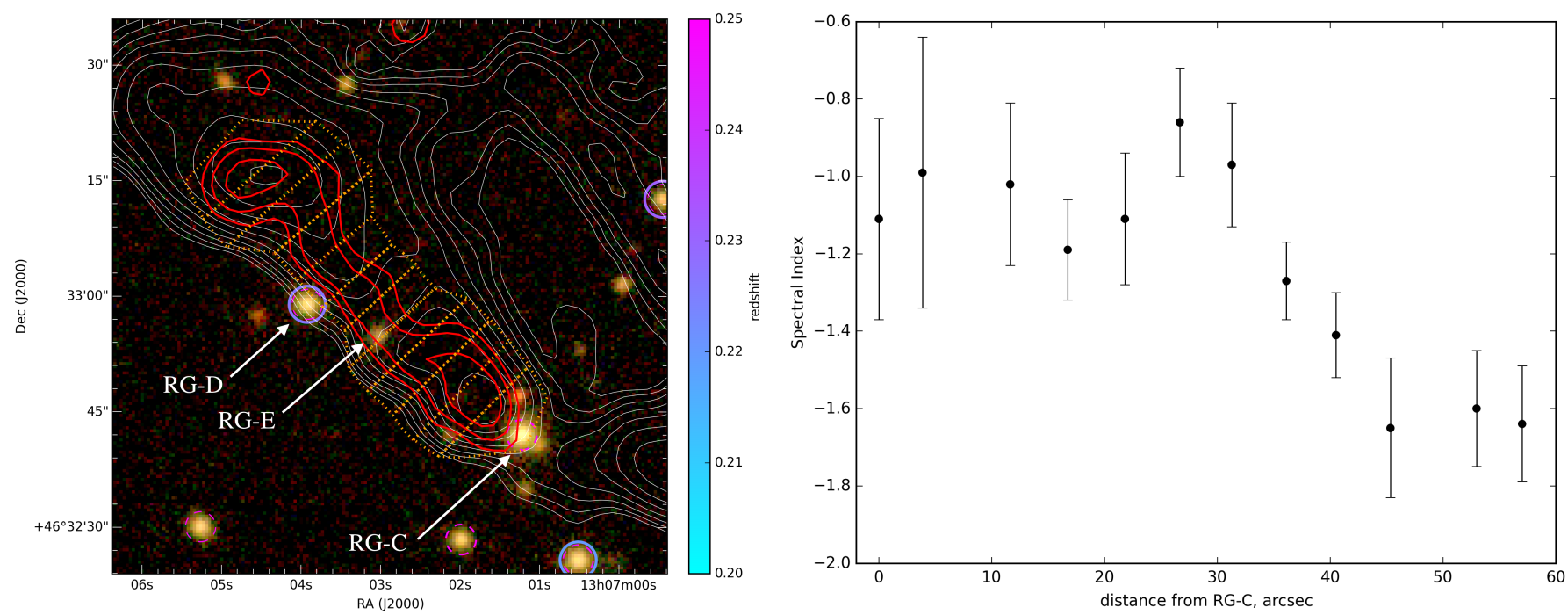

Fig. 9. Left: zoom in on radio galaxy candidates RG-C, -D, and -E. LOFAR contours are in white, GMRT contours in red, both at 6" resolution starting at five times the rms noise $\left(90 \mu \mathrm{Jy} \mathrm{beam}^{-1}\right)$ and increasing by a factor of 1.4. Orange dashed lines mark the position of spectral index measurements. Right: spectral index as a function of distance from RG-C. Measurements are an average of all pixels in the region, taking all emission above five times the rms noise.

The spectral index information from our LOFAR $150 \mathrm{MHz}$ observation to our reprocessed GMRT observation at $610 \mathrm{MHz}$ (Fig. 7) shows very little variation along the length and width of the NW ridge. The steepest part is at the start of the ridge near BCG-B with a spectral index of $\alpha=-1.96 \pm 0.16$. In the middle of the ridge the spectral index flattens to $\alpha=-1.1 \pm 0.15$ (which is coincident with various optical counterparts in Fig. 6 that could host AGNs). Near the end of the ridge farthest from BCG-B, the spectral index is $\alpha=-1.45 \pm 0.17$. Overall, we see that the NW ridge produces very steep spectrum emission, without any significant gradient along its length other than when it connects with BCG-B, becoming $\alpha=-0.65 \pm 0.15$. This jump to flatter spectral index values near BCG-B indicates its AGN has more recently restarted. Whilst we do not observe a spectral index gradient perpendicular to the length of the ridge either (as seen in Venturi et al. 2013), we do see the LOFAR emission at $150 \mathrm{MHz}$ extends farther southwest than the GMRT emission at $610 \mathrm{MHz}$, indicating the spectrum steepens in this direction. If the NW ridge is a radio relic this extra steep spectrum emission seen by LOFAR would be expected if formed from a shock moving northeast, as proposed in Venturi et al. (2013). However, the same effect could also be achieved by the radio lobe expanding to the east, or if BCG-B has moved northeast with its radio lobe, leaving older and steeper spectrum emission to the southwest of the NW ridge as we see with LOFAR.

If the NW ridge was re-accelerated in a radio-relic-like scenario, its position is atypical, being perpendicular to the cluster merger axis. In a simplified geometrical set up assuming the relic is viewed side-on, it would be expected that a shock front is moving from southwest to northeast, which is counterintuitive to the cluster merger axis of southeast to northwest. Furthermore, compressed, denser X-ray gas should be found to the southwest. In the X-ray image (Fig. 8) there is no indication of a jump in surface brightness coinciding with the NW ridge. In simulations of radio-relic formation there are cases of atypical relics in complex merger scenarios, such as object A in Fig. 7 of Nuza et al. (2017). The NW ridge in A1682 is similar to this, and it could be the case that A1682 is one of the few examples of relics which are not caused by a peripheral, spherical shock.
The NW ridge also extends hundreds of kiloparsecs farther to the northwest than previous observations, and with the nondetection in the GMRT data in this region, we infer that this is an area of very steep spectrum emission, constraining the spectral index as $\alpha<-2.5$ given the non-detection with the GMRT. Given that this emission reaches up to $0.6 \mathrm{Mpc}$ from BCG-B, if it originated from BCG-B it is likely to be very old emission with a very steep spectral index.

Overall, we believe the most likely origin of the NW ridge is emission from a previous epoch of AGN activity (an old radio lobe) associated with BCG-B. The jump in spectral index from steeper to flatter values around BCG-B is likely caused by the AGN in BCG-B having more recently restarted. This emission over the NW ridge has the potential to have been re-accelerated by the merger event in the region closer to the centre of the cluster, although we find no conclusive evidence given this data.

\subsection{The diffuse component}

The diffuse component identified in previous studies as an USSRH candidate is resolved in this new observation as a feature distinct from the SE ridge. The resolved radio emission traced by the solid cyan lines in Fig. 4 is morphologically indicative of a wide-angle tailed (WAT; Sakelliou \& Merrifield 2000) radio galaxy. A likely optical counterpart identified in SDSS is RG-B (13:06:54.34, +46:32:30.46). If so, this source could be moving southwest where its radio-loud AGN has left the observed radio structures in its wake. The extension of the radio lobes to the northeast could also be due to the jet propagation at relativistic speeds and the cluster environment influencing their morphology. The source RG-B looks to be a merging galaxy, and has a photometric redshift in SDSS of $0.293 \pm 0.063$. Despite the general bias towards higher redshifts for photometrically observed galaxies it is highly likely to be a cluster member. The region where the two cyan lines join to create the very-lowbrightness tail (traced by a dashed cyan line) could represent a period where the radio jets from the AGN were of much lower power or have been more confined by the ICM, only creating a single traced line of emission (often referred to as narrow-angle tailed radio galaxies; Stocke \& Burns 1987). 
If the emission highlighted by cyan lines is indeed associated with RG-B then its spectral properties in the radio emission make it difficult to deduce a precise evolutionary scenario. Previous studies show this emission has a very steep spectrum, with none of the emission detected by Venturi et al. (2008) in their deep GMRT observations at $610 \mathrm{MHz}$. Given our LOFAR observations and their non-detection we expect a spectral index of $\alpha<-2.5$. If RG-B is currently (or has recently been) active, it is expected that emission near the proposed optical host should have a much flatter spectrum. It therefore appears clear that the AGN in RG-B is no longer radio loud and has been inactive for a significant period of time. Such sources are referred to as remnant radio galaxies which typically have ages of around a few hundred million years (e.g. Brienza et al. 2017; Murgia et al. 2011). It therefore seems most likely that RG-B has been sitting more or less where it currently is for approximately this period of time since it was last active, and has not moved from northwest to southeast, unless its motion has been halted in its current location for a significant period of time. Instead, the propagation of the jets, along with the influence from the ICM, has created the morphology of the radio structures observed in this area.

Whilst RG-B is the most compelling candidate for the diffuse component in terms of its location, there are numerous optical counterparts in the region (all are likely cluster members) which could also have contributed to the radio emission. If the host was moving from northeast to southwest, it is likely to be one of the sources farther to the southwest where there is no observed radio emission, and not RG-B.

Overall, the very steep spectrum emission in this area of the cluster is likely a result of significant synchrotron ageing from very old radio galaxy emission. Within a galaxy cluster it has been proposed that remnant radio sources can be observed for longer than would be expected due to lower adiabatic losses since the dense cluster environment prevents the radio lobes expanding as much (Murgia et al. 2011). It could also be the case that re-acceleration mechanisms as a result of the cluster merger have also contributed to highlighting this remnant source with such a steep spectrum. However, without detailed modelling over a wide bandwidth (such as analysis done by de Gasperin et al. 2017), it is not possible to distinguish between ageing electrons in an old radio galaxy tail, and re-acceleration.

\subsection{Searching for a ultra-steep-spectrum radio halo}

In previous studies the emission labelled as the diffuse component was classified as a potential USSRH, but as discussed our highresolution LOFAR images show that this is unlikely. Instead we investigate the area between the diffuse component and the BCGs as a potential USSRH (region A in Fig. 5). This area is closer to the centre of the X-ray emission from the cluster (see Fig. 8), where re-acceleration mechanisms as a result of the merger event will be more powerful. The low resolution of previous spectral studies prevents this area from being distinguished from the diffuse component. Our LOFAR observations show the presence of significant residuals in this area at 6" resolution (see Figs. 4 and 6), and direct detection of significantly extended emission at $20^{\prime \prime}$ resolution (see Figs. 5 and 6). This suggests that particle acceleration has occurred in this area over large spatial scales (up to $500 \mathrm{kpc}$ ). The diffuse and low-surface-brightness emission encompassing region A is unlikely to be connected with any AGN identified in the high-resolution image, and is in a location between the two sub-clusters (close to the centre of the X-ray emission; Fig. 8) which supports an origin from the re-acceleration of electrons in the wake of a merger event.
We re-imaged the $610 \mathrm{MHz}$ GMRT data at a resolution of $20^{\prime \prime}$ (the same as our LOFAR map in Fig. 5) reaching an rms noise of $280 \mu \mathrm{Jy}$, showing no emission in region A. Given the non-detection of emission in this region at $610 \mathrm{MHz}$, we show this is relatively steep spectrum emission with a spectral index of $\alpha<-1.1$, which is a signature of re-acceleration models (Brunetti et al. 2008; Brunetti \& Jones 2014). However, without spectral modelling over a wide bandwidth we cannot tell between re-acceleration and the ageing of fossil radio emitting plasma which could be left over from old radio galaxies (Enßlin \& Gopal-Krishna 2001). Overall we conclude that region A is very likely to be a radio halo caused by the merger event. The presence of many AGNs with radio jets throughout the cluster presents an obvious mechanism to fill the ICM with a seed population of relativistic electrons to fuel this radio halo emission.

\subsection{Additional diffuse emission}

Other areas of extended diffuse emission are located around the cluster, as labelled in Fig. 5. Whilst region A is the strongest, regions $\mathrm{B}, \mathrm{C}$, and $\mathrm{D}$ also show significant diffuse emission not seen before and without significant detection in the 6" image. These regions could be very old and mildly relativistic electrons left over from the lobes of radio galaxies, with the possibility that they have been re-accelerated by the cluster merger event. Region B could be old emission from the lobes of a radio galaxy moving southeast associated with RG-A or BCG-A and the dashed green lines in Fig. 4. Region C could also be associated with this, but appears to be disconnected, so instead could be an extension of the NW ridge. In region D there are numerous radio loud galaxies and candidates (shown in Fig. 6) which could have had radio lobes that contributed to this emission.

Region $\mathrm{E}$ is distinct from the rest of the cluster. It shows minimal emission in the 6 " resolution image, and is only significantly detected in the $20^{\prime \prime}$ resolution image. There is one photometrically identified cluster member to the northeast of this region with a radio loud AGN (dashed cyan circle in Fig. 6), though it is disconnected from region $\mathrm{E}$. There is a bright galaxy in the centre of region $\mathrm{E}$ with the appearance of an orange elliptical (13:06:27.84, $+46: 36: 40.14)$ which has a photometric redshift of $0.302 \pm 0.047$. It has a slightly disturbed morphology indicating that it is undergoing a merger event. This could be the host of a remnant radio galaxy currently with no radio-loud AGN, where its lobes have aged, becoming very faint and diffuse. Its extended radio structure is measured at $154 \pm 10 \mathrm{arcsec}$, giving this giant remnant radio galaxy a physical size of $1.29 \pm$ $0.29 \mathrm{Mpc}$ (where the error in the photometric redshift dominates). It has an integrated flux density of $30.6 \pm 6.1 \mathrm{mJy}$, giving a luminosity of $9.61_{-2.40}^{+6.49} \times 10^{24} \mathrm{~W} \mathrm{~Hz}^{-1}$ at $150 \mathrm{MHz}$. Given the observed bias towards higher values for photometric redshifts of cluster members, we also calculate its physical size based on the average redshift of the cluster $(z=0.225)$, as $0.86 \pm 0.06 \mathrm{Mpc}$, giving a luminosity of $2.83 \pm 1.02 \times 10^{24} \mathrm{~W} \mathrm{~Hz}^{-1}$. Whilst region $\mathrm{E}$ is elongated and aligned perpendicular to the cluster merger axis and morphologically indicative of a radio relic, it is more than $1 \mathrm{Mpc}$ away from the cluster centre at the redshift of the cluster (and with an optical host at a higher redshift), making it very unlikely to be a relic.

\subsection{Giant radio galaxies}

There are numerous extended radio sources observed surrounding the galaxy cluster which are likely background sources (AGNs with radio jets and lobes) at higher redshift. Many of 
them do not have discernible optical counterparts in the SDSS image; however, we note a handful of them which do and present them as giant radio galaxies with both Fanaroff-Riley type I and II (FR-I, FR-II; Fanaroff \& Riley 1974) morphology.

In Fig. 10 we present two new radio galaxies to the north of the cluster. The radio galaxy in the left of this figure has an optical host identified in SDSS (13:06:17.78, +46:45:59.77) with an SDSS $r$-band magnitude of 21.86 and a photometric redshift of $0.615 \pm 0.060$. The extended radio structure is measured at $156 \pm 10 \mathrm{arcsec}$, giving this giant radio galaxy a physical size of $2.86 \pm 0.55 \mathrm{Mpc}$ (combined errors from angular size and redshift). There are three other galaxies situated between and in line with the radio lobes which are less likely candidates as optical hosts but could be part of a galaxy group ( $a$ : 13:06:17.45, +46:46:01.59, $b:$ 13:06:16.64, +46:46:04.31, $c$ : 13:06:16.12, +46:46:06.66). These lie 12, 18, and 25 arcsec to the west of the proposed optical host, respectively, with SDSS $r$ band magnitudes of $24.41 \pm 0.54,22.98 \pm 0.20$, and $22.96 \pm 0.30$. Candidates $a$ and $c$ have photometric redshifts of $0.879 \pm 0.1443$ and $0.696 \pm 0.1108$, respectively. This giant radio galaxy has a distinct FR-II morphology with two separate lobes. The left lobe has an integrated flux density of $47.5 \pm 9.5 \mathrm{mJy}$ at $150 \mathrm{MHz}$, and $8.5 \pm 1.7 \mathrm{mJy}$ at $610 \mathrm{MHz}$. The average spectral index of the left lobe is $\alpha=-0.74 \pm 0.32$ with a standard deviation of 0.32 . The right lobe has an integrated flux density of $43.9 \pm 8.8 \mathrm{mJy}$ at $150 \mathrm{MHz}$, and $8.0 \pm 1.6 \mathrm{mJy}$ at $610 \mathrm{MHz}$. The average spectral index of the right lobe is $\alpha=-0.74 \pm 0.37$ with a standard deviation of 0.24 . In total its luminosity is $1.56_{-0.58}^{+0.79} \times 10^{26} \mathrm{~W} \mathrm{~Hz}^{-1}$ at $150 \mathrm{MHz}$.

The radio galaxy in the right of Fig. 10 has an optical host identified in SDSS (13:05:47.89, +46:47:28.85) with an SDSS $r$-band magnitude of $20.34 \pm 0.03$, and photometric redshift of $0.415 \pm 0.035$. The extended radio structure is measured at $60 \pm 10 \mathrm{arcsec}$, giving this radio galaxy a physical size of $0.68 \pm 0.20 \mathrm{Mpc}$. This radio galaxy has an FR-II morphology with two connected lobes. The left lobe has an integrated flux density of $23.9 \pm 4.8 \mathrm{mJy}$ at $150 \mathrm{MHz}$, and $10.0 \pm 2.0 \mathrm{mJy}$ at $610 \mathrm{MHz}$. The average spectral index of the left lobe is $\alpha=$ $-0.50 \pm 0.31$ with a standard deviation of 0.24 . The right lobe has an integrated flux density of $49.2 \pm 9.8 \mathrm{mJy}$ at $150 \mathrm{MHz}$, and $20.9 \pm 4.2 \mathrm{mJy}$ at $610 \mathrm{MHz}$. The average spectral index of the right lobe is $\alpha=-0.52 \pm 0.25$ with a standard deviation of 0.31 . Considering the large size of the radio lobes these spectral index values are flatter than what would normally be expected, although they are consistent within the errors. In total its luminosity is $4.84_{-1.70}^{+2.20} \times 10^{25} \mathrm{~W} \mathrm{~Hz}^{-1}$ at $150 \mathrm{MHz}$ (where the error is dominated by the uncertainty in redshift).

In the left of Fig. 11 there are two radio galaxies (also seen in the southeast of Fig. 6) in the periphery of Abell 1682. The radio galaxy in the north of this figure is coincident with a bright foreground star, but has an optical host identified in SDSS (13:07:20.83, +46:30:52.67) that is well positioned in-line with the radio lobes (shown by a yellow circle). This host has an SDSS $r$-band magnitude of $21.42 \pm 0.10$ and spectroscopic redshift of $0.6038 \pm 0.0002$. The extended radio structure is measured at $116 \pm 10 \mathrm{arcsec}$, giving this giant radio galaxy a physical size of $2.07 \pm 0.18 \mathrm{Mpc}$ (where the error in angular size dominates and redshift error is negligible). There is another galaxy situated 5 arcsec to the southeast (13:07:21.43, $+46: 30: 49.36$ ) which is off-centre from the radio structure but is likely to be a part of the same galaxy group. This has a spectroscopic redshift of $0.6025 \pm 0.0002$ and an SDSS $r$-band magnitude of $20.41 \pm 0.08$. This giant radio galaxy has an FR-I morphology, with an integrated flux density of $186.1 \pm 28.0 \mathrm{mJy}$ at
$150 \mathrm{MHz}$, and $46.3 \pm 9.3 \mathrm{mJy}$ at $610 \mathrm{MHz}$. This gives a luminosity of $3.04 \pm 0.61 \times 10^{26} \mathrm{~W} \mathrm{~Hz}^{-1}$ at $150 \mathrm{MHz}$. The average spectral index is $\alpha=-0.80 \pm 0.23$ with a standard deviation of 0.47 .

The radio galaxy in the southwest of Fig. 11 has an optical host identified in SDSS (13:07:18.07, +46:29:46.61) that is positioned in the centre of the radio lobes (shown by green circle). It has an SDSS $r$-band magnitude of $20.82 \pm 0.08$ and spectroscopic redshift of $0.7416 \pm 0.0002$. The extended radio structure is measured at $55 \pm 10$ arcsec from east to west, giving this giant radio galaxy a physical size of $1.27 \pm 0.22 \mathrm{Mpc}$. This giant radio galaxy has an FR-I morphology, with an integrated flux density of $79.4 \pm 15.9 \mathrm{mJy}$ at $150 \mathrm{MHz}$, and $24.2 \pm 4.8 \mathrm{mJy}$ at $610 \mathrm{MHz}$. This gives a luminosity of $2.15 \pm 0.42 \times 10^{26} \mathrm{~W} \mathrm{~Hz}^{-1}$ at $150 \mathrm{MHz}$. The average spectral index is $\alpha=-0.60 \pm 0.25$ with a standard deviation of 0.62 .

In the right of Fig. 11 the only potential optical host with a redshift measurement in SDSS is circled in yellow (13:07:54.32, $+46: 45: 11.92)$. Whilst it is slightly off-centre towards the east lobe, it is one of the most likely host galaxy candidates in the area. It has a photometric redshift of $0.409 \pm 0.104$, and an $r$-band magnitude of $22.42 \pm 0.14$. There is another optical source 1 arcsec to the southwest just outside of the radio emission which has no redshift measurement. This source is classified as a star in SDSS, although it could well be a high-redshift quasar. The extended radio structure is measured at $96 \pm 5$ arcsec, giving this giant radio galaxy a physical size of $1.08 \pm 0.36 \mathrm{Mpc}$ (where the error in redshift dominates). It has an FR-II morphology, where the left lobe has an integrated flux density of $24.3 \pm 4.9 \mathrm{mJy}$ at $150 \mathrm{MHz}$, and the right lobe has an integrated flux density of $12.1 \pm 2.4 \mathrm{mJy}$. In total this gives a luminosity of $2.33_{-1.40}^{+2.47} \times 10^{25} \mathrm{~W} \mathrm{~Hz}^{-1}$ at $150 \mathrm{MHz}$ (where the error is dominated by the uncertainty in redshift). There is a negligible detection with the GMRT at $610 \mathrm{MHz}$.

\section{Conclusions}

Deep radio observations at $150 \mathrm{MHz}$ using LOFAR have built on previous knowledge to provide a clearer picture of the phenomena in Abell 1682 as well as detecting new emission not seen before. Given the complexity of the radio emission we detect, deducing a precise dynamical scenario for this cluster and the origin of all the emission is extremely difficult.

At $6^{\prime \prime}$ resolution new filamentary structures are observed up to $600 \mathrm{kpc}$ in length. Many of these are proposed to be the jets and lobes from old radio galaxies which are moving through the cluster. Both BCGs are seen to have extended radio features associated with them, with the small extension of BCG-A having not been seen before. We present a radio spectral index map covering the range $150-610 \mathrm{MHz}$ (through a re-analysis of archival GMRT data), showing that the emission is mainly steep spectrum (up to $\alpha=-2.1 \pm 0.2$ ), with flatter spectrum emission (with $\alpha=-0.65 \pm 0.20$ ) typical of AGN activity around both BCGs. The extended radio structure connected to BCG-B (labelled the NW ridge) shows no gradient in the spectral index along its length or width, but is significantly more extended in our $150 \mathrm{MHz}$ observations compared to observations at $610 \mathrm{MHz}$. Given the non-detection of this extension by archival observations at higher frequencies we place a limit on the spectral index of $\alpha<-2.5$. Its features and orientation are unlike a typical radio relic, however there is the possibility that the cluster merger event has contributed to the emission mechanisms by re-accelerating the electrons through a low Mach number shock. Overall it is most likely that the $\mathrm{N}-\mathrm{W}$ ridge is an old radio lobe from AGN activity in BCG-B. 

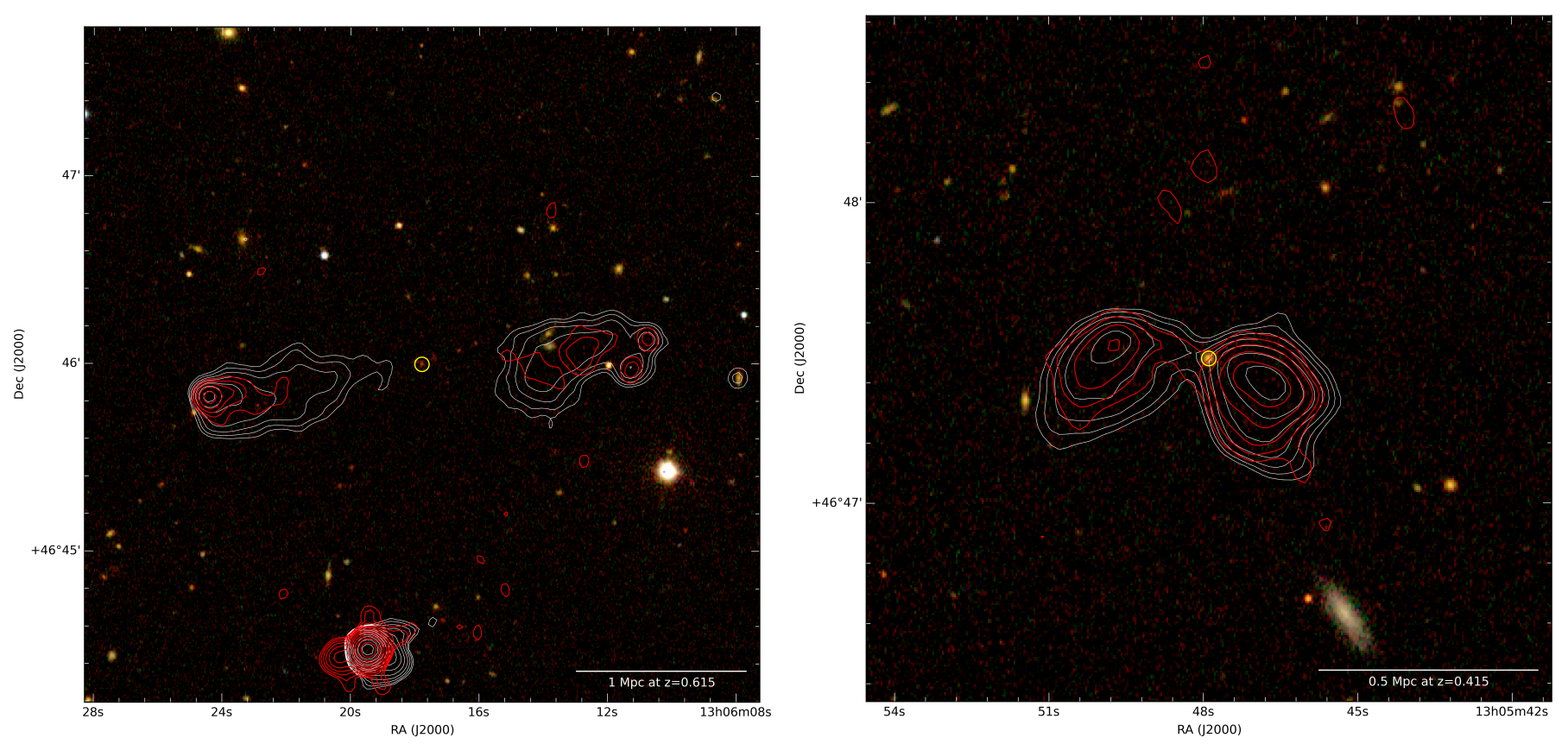

Fig. 10. Left: giant radio galaxy with the likely optical host circled in yellow, identified in SDSS (13:06:17.78, +46:45:59.77) with a photometric redshift of $0.615 \pm 0.060$. This giant radio galaxy has a physical size of $2.86 \pm 0.55 \mathrm{Mpc}$. Right: giant radio galaxy with the likely optical host circled in yellow, identified in SDSS (13:05:47.89, $+46: 47: 28.85)$ with a photometric redshift of $0.415 \pm 0.035$. This radio galaxy a physical size of $0.68 \pm 0.20 \mathrm{Mpc}$. In both images the background is an SDSS composite image from bands $g, r$, and $i$. LOFAR and GMRT contours are overlaid at $6^{\prime \prime}$ resolution starting at five times the rms noise $\left(90 \mu \mathrm{Jy}_{\text {beam }}^{-1}\right)$, and increase by a factor of 1.6 .
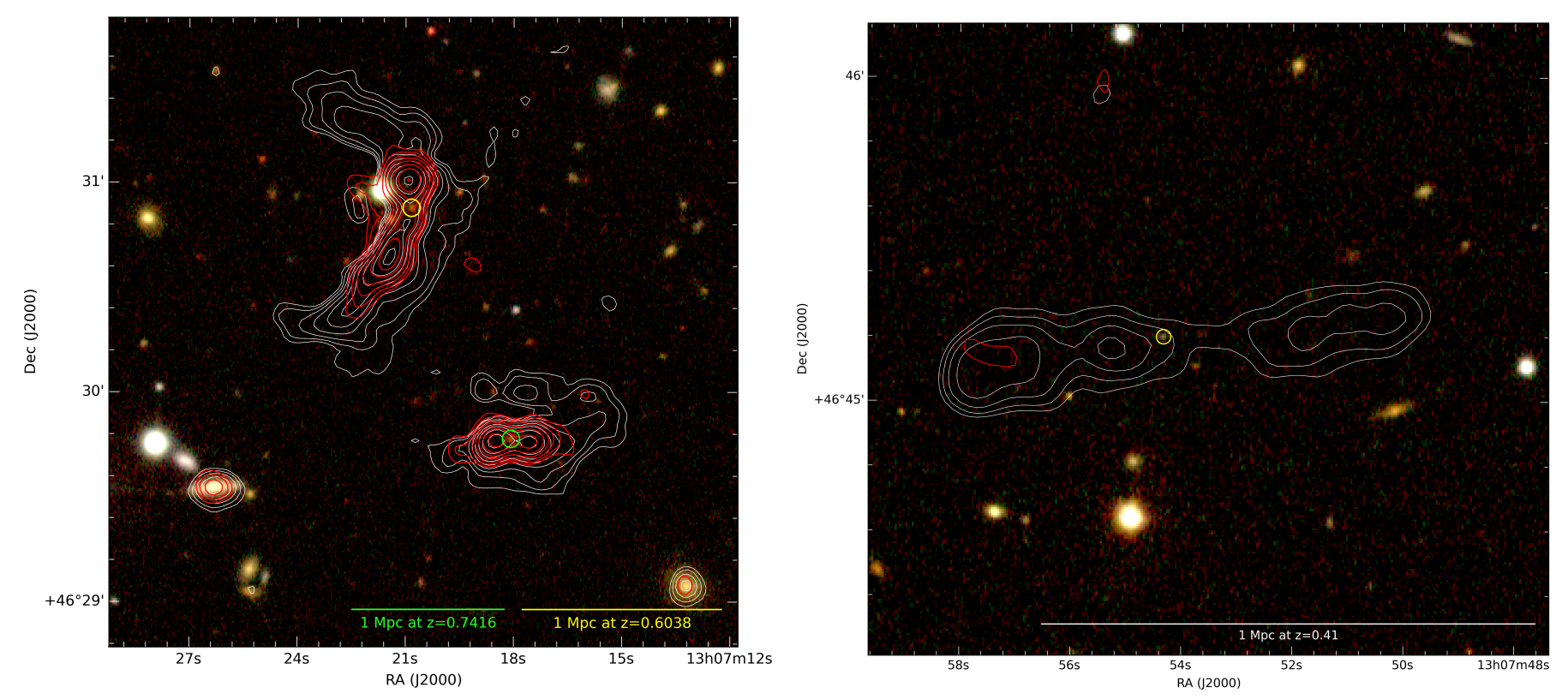

Fig. 11. Left: two giant radio galaxies with likely optical hosts circled. In yellow (13:07:20.83, +46:30:52.67) the host galaxy has a spectroscopic redshift of $0.6038 \pm 0.0002$. This giant radio galaxy has a physical size of $2.07 \pm 0.18 \mathrm{Mpc}$. In green $(13: 07: 18.07,+46: 29: 46.61)$ the host galaxy has a spectroscopic redshift of $0.7416 \pm 0.0002$. This giant radio galaxy a physical size of $1.27 \pm 0.22 \mathrm{Mpc}$ measured from east to west. Right: giant radio galaxy with a potential optical host circled in yellow, identified in SDSS (13:07:54.32, $+46: 45: 11.92)$ with a photometric redshift of $0.409 \pm 0.104$. It has a physical size of $1.08 \pm 0.36 \mathrm{Mpc}$. In both images the background is an SDSS composite image from bands $g, r$, and $i$. LOFAR and GMRT contours are overlaid at $6^{\prime \prime}$ resolution starting at five times the rms noise $\left(90 \mu \mathrm{Jy} \mathrm{beam}^{-1}\right)$, and increase by a factor of 1.6 .

Furthermore, the large radio structure extending from BCG-B to the northeast is likely to be the second lobe from the AGN in BCG-B.

The area in the southeast of the cluster previously identified as an USSRH is resolved into a component strongly resembling the tail from a radio galaxy moving southeast through the cluster, though this is mainly a morphological classification since there is no redshift for the optical counterpart or spectral index information on the host. Given that none of this emission is observed in archival GMRT observations at $610 \mathrm{MHz}$ we place a limit on the spectral index of $\alpha<-2.5$. A separate component in the southeast is resolved and also detected at $610 \mathrm{MHz}$, showing a typical 


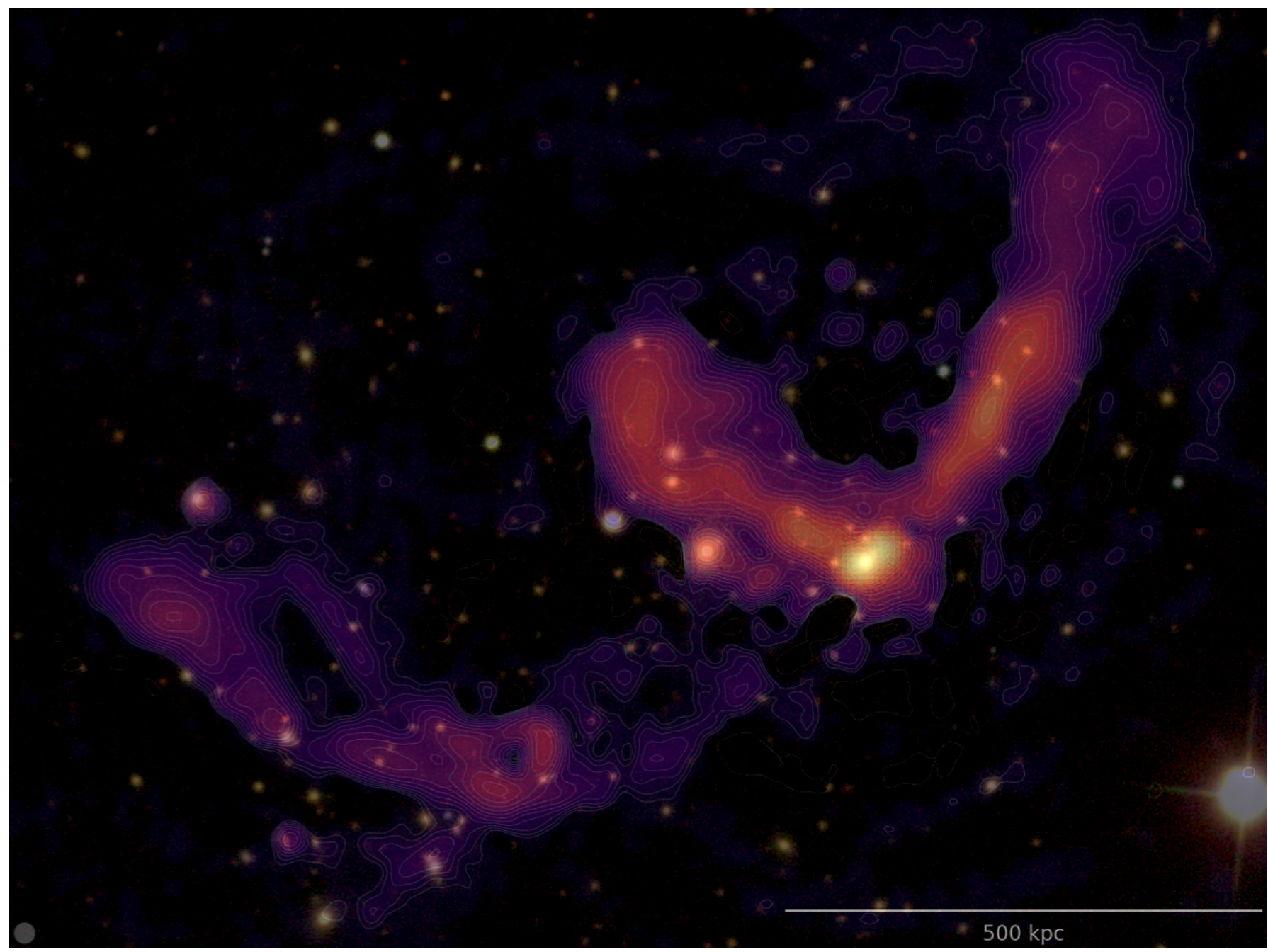

Fig. 12. LOFAR radio image and contours at a resolution of at 6" (as shown in Fig. 4) overlaid on the optical background from SDSS.

spectral index profile of a radio galaxy, but without a conclusive optical host for its AGN.

At $20^{\prime \prime}$ resolution significant diffuse emission is discovered throughout the cluster. We believe most of these areas in the outskirts of the cluster are a result of radio lobes expanding into less-dense regions of the ICM, or from remnant plasma left over as radio galaxies have moved through the cluster. The area between the centres of mass of the two sub-clusters is bridged by steep-spectrum ( $\alpha<-1.1$ given the non-detection in archival $610 \mathrm{MHz}$ GMRT observations) low-surface-brightness emission over hundreds of kiloparsecs resembling that of a radio halo. This strongly indicates that re-acceleration mechanisms are in play as a result of the cluster merger event. The presence of AGNs throughout the cluster present a compelling mechanism for filling the ICM with relativistic electrons capable of fuelling the radio halo emission after the cluster merger event.

Five new giant radio galaxies are observed in the field of Abell 1682 where we find optical hosts with either photometric or spectroscopic redshifts in SDSS (that are not cluster members). They have physical sizes and luminosities (at $150 \mathrm{MHz}$ ) of: $2.86 \pm 0.55 \mathrm{Mpc}, 1.56_{-0.53}^{+0.69} \times 10^{26} \mathrm{~W} \mathrm{~Hz}^{-1}$ (FR-II); $0.68 \pm 0.20 \mathrm{Mpc}, 4.84_{-1.50}^{+1.91} \times 10^{25} \mathrm{~W} \mathrm{~Hz}^{-1}$ (FR-II); $2.07 \pm 0.18 \mathrm{Mpc}$, $3.04 \pm 0.46 \times 10^{26} \mathrm{~W} \mathrm{~Hz}^{-1}$ (FR-I); $1.27 \pm 0.22 \mathrm{Mpc}, 24.2 \pm 3.6 \mathrm{mJy}$ (FR-I); and $1.08 \pm 0.36 \mathrm{Mpc}, 2.33_{-1.34}^{+2.28} \times 10^{25} \mathrm{~W} \mathrm{~Hz}^{-1}$ (FR-II). Furthermore, we find a giant remnant radio galaxy on the out- skirts of Abell 1682 (associated with a cluster member) with a physical size of $1.29 \pm 0.29 \mathrm{Mpc}$, and a luminosity of $9.61_{-2.40}^{+6.49} \times$ $10^{24} \mathrm{~W} \mathrm{~Hz}^{-1}$.

Acknowledgements. We thank the referee for providing valuable comments helping to improve the clarity and interpretation of the science in this paper. A.O.C gives thanks to Ben Stappers for providing extensive comments on this paper as part of his $\mathrm{PhD}$ thesis. A.O.C, A.M.M.S, and T.M.C gratefully acknowledge support from the European Research Council under grant ERC2012-StG-307215 LODESTONE. A.B gratefully acknowledges support from the European Research Council under grant ERC-StG-714245 DRANOEL, n. 714245 , and from the MIUR grant FARE SMS. RJvW acknowledges support from the ERC Advanced Investigator programme NewClusters 321271 and the VIDI research programme with project number 639.042.729, which is financed by the Netherlands Organisation for Scientific Research (NWO). LOFAR, the Low Frequency Array designed and constructed by ASTRON, has facilities in several countries that are owned by various parties (each with their own funding sources), and that are collectively operated by the International LOFAR Telescope (ILT) foundation under a joint scientific policy. This research has made use of the NASA/IPAC Extragalactic Database (NED) which is operated by the Jet Propulsion Laboratory, California Institute of Technology, under contract with the National Aeronautics and Space Administration. This research made use of Numpy (Oliphant 2006), APLpy (an open-source plotting package for Python; Robitaille \& Bressert 2012), Matplotlib (Hunter 2007), and the cosmology calculator published in Wright (2006). Funding for the Sloan Digital Sky Survey IV has been provided by the Alfred P. Sloan Foundation, the U.S. Department of Energy Office of Science, and the Participating Institutions. SDSS-IV acknowledges support and resources from the Center for HighPerformance Computing at the University of Utah. The SDSS web site is WwW.sdss.org. SDSS-IV is managed by the Astrophysical Research 
Consortium for the Participating Institutions of the SDSS Collaboration including the Brazilian Participation Group, the Carnegie Institution for Science, Carnegie Mellon University, the Chilean Participation Group, the French Participation Group, Harvard-Smithsonian Center for Astrophysics, Instituto de Astrofísica de Canarias, The Johns Hopkins University, Kavli Institute for the Physics and Mathematics of the Universe (IPMU)/University of Tokyo, Lawrence Berkeley National Laboratory, Leibniz Institut für Astrophysik Potsdam (AIP), Max-Planck-Institut für Astronomie (MPIA Heidelberg), Max-Planck-Institut für Astrophysik (MPA Garching), Max-Planck-Institut für Extraterrestrische Physik (MPE), National Astronomical Observatory of China, New Mexico State University, New York University, University of Notre Dame, Observatário Nacional/MCTI, The Ohio State University, Pennsylvania State University, Shanghai Astronomical Observatory, United Kingdom Participation Group, Universidad Nacional Autónoma de México, University of Arizona, University of Colorado Boulder, University of Oxford, University of Portsmouth University of Utah, University of Virginia, University of Washington, University of Wisconsin, Vanderbilt University, and Yale University.

\section{References}

Albareti, F. D., Allende Prieto, C., Almeida, A., et al. 2017, ApJS, 233, 25 Allen, S. W., Edge, A. C., Fabian, A. C., et al. 1992, MNRAS, 259, 67

Beck, R., Dobos, L., Budavári, T., Szalay, A. S., \& Csabai, I. 2016, MNRAS 460, 1371

Blandford, R., \& Eichler, D. 1987, Phys Rep., 154, 1

Botteon, A., Gastaldello, F., Brunetti, G., \& Dallacasa, D. 2016, MNRAS, 460, L84

Botteon, A., Shimwell, T. W., Bonafede, A., et al. 2018, MNRAS, 478, 885

Brienza, M., Godfrey, L., Morganti, R., et al. 2017, A\&A, 606, A98

Briggs, D. S. 1995, PhD Thesis, New Mexico Institute of Mining Technology, Socorro, USA

Brunetti, G., \& Jones, T. W. 2014, Int. J. Mod. Phys. D, 23, 1430007

Brunetti, G., \& Lazarian, A. 2007, MNRAS, 378, 245

Brunetti, G., \& Lazarian, A. 2011, MNRAS, 410, 127

Brunetti, G., Setti, G., Feretti, L., \& Giovannini, G. 2001, MNRAS, 320, 365

Brunetti, G., Giacintucci, S., Cassano, R., et al. 2008, Nature, 455, 944

Cassano, R., Brunetti, G., \& Setti, G. 2006, MNRAS, 369, 1577

Dahle, H., Kaiser, N., Irgens, R. J., Lilje, P. B., \& Maddox, S. J. 2002, ApJS, 139,313

de Gasperin, F. 2017, MNRAS, 467, 2234

de Gasperin, F., Intema, H. T., Shimwell, T. W., et al. 2017, Sci. Adv., 3, e1701634

Ebeling, H., Edge, A. C., Bohringer, H., et al. 1998, MNRAS, 301, 881

Eckert, D., Jauzac, M., Vazza, F., et al. 2016, MNRAS, 461, 1302

Eisenstein, D. J., Weinberg, D. H., Agol, E., et al. 2011, ApJ, 142, 72

Ensslin, T. A., Biermann, P. L., Klein, U., \& Kohle, S. 1998, A\&A, 332, 395

Enßlin, T. A., \& Gopal-Krishna 2001, A\&A, 366, 26

Fanaroff, B. L., \& Riley, J. M. 1974, MNRAS, 167, 31P

Flewelling, H. A., Magnier, E. A., Chambers, K. C., et al. 2016, ArXiv e-prints [arXiv:1612.05243]

Guo, X., Sironi, L., \& Narayan, R. 2014a, ApJ, 794, 153

Guo, X., Sironi, L., \& Narayan, R. 2014b, ApJ, 797, 47
Harwood, J. J., Hardcastle, M. J., Croston, J. H., \& Goodger, J. L. 2013, MNRAS, 435, 3353

Harwood, J. J., Hardcastle, M. J., \& Croston, J. H. 2015, MNRAS, 454, 3403

Hoang, D. N., Shimwell, T. W., Stroe, A., et al. 2017, MNRAS, 471, 1107

Hunter, J. D. 2007, Comput. Sci. Eng., 9, 90

Intema, H. T. 2014, Astron. Soc. India Conf. Ser., 13, 469

Intema, H. T., Jagannathan, P., Mooley, K. P., \& Frail, D. A. 2017, A\&A, 598, A78

Lane, W. M., Cotton, W. D., van Velzen, S., et al. 2014, MNRAS, 440, 327

Macario, G., Venturi, T., Intema, H. T., et al. 2013, A\&A, 551, A141

Mohan, N., \& Rafferty, D. 2015, Astrophysics Source Code Library [record ascl:1502.007]

Murgia, M., Parma, P., Mack, K. H., et al. 2011, A\&A, 526, A148

Nuza, S. E., Gelszinnis, J., Hoeft, M., \& Yepes, G. 2017, MNRAS, 470, 240

Oliphant, T. 2006, NumPy: A Guide to NumPy (USA: Trelgol Publishing)

Pandey, V. N., van Zwieten, J. E., de Bruyn, A. G., \& Nijboer, R. 2009, in The

Low-Frequency Radio Universe, eds. D. J. Saikia, D. A. Green, Y. Gupta, \&

T. Venturi, ASP Conf. Ser., 407, 384

Petrosian, V. 2001, ApJ, 557, 560

Pinzke, A., Oh, S. P., \& Pfrommer, C. 2015, ArXiv e-prints [arXiv:1503. 07870]

Pinzke, A., Oh, S. P., \& Pfrommer, C. 2017, MNRAS, 465, 4800

Planck Collaboration XXXII. 2015, A\&A, 581, A14

Planck Collaboration XXVII. 2016, A\&A, 594, A27

Planck Collaboration XIII. 2016, A\&A, 594, A13

Robitaille, T., \& Bressert, E. 2012, Astrophysics Source Code Library [record ascl:1208.017]

Sakelliou, I., \& Merrifield, M. R. 2000, MNRAS, 311, 649

Scaife, A. M. M., \& Heald, G. H. 2012, MNRAS, 423, L30

Shimwell, T. W., Luckin, J., Brüggen, M., et al. 2016, MNRAS, 459, 277

Shimwell, T. W., Tasse, C., Hardcastle, M. J., et al. 2019, A\&A, 622, A1

Smirnov, O. M., \& Tasse, C. 2015, MNRAS, 449, 2668

Stocke, J. T., \& Burns, J. O. 1987, ApJ, 319, 671

Stroe, A., Harwood, J. J., Hardcastle, M. J., \& Röttgering, H. J. A. 2014, MNRAS, 445, 1213

Swarup, G. 1991, in IAU Colloq. 131: Radio Interferometry. Theory, Techniques, and Applications, eds. T. J. Cornwell, \& R. A. Perley, ASP Conf. Ser., 19, 376 Tasse, C. 2014, ArXiv e-prints [arXiv:1410.8706]

Tasse, C., Hugo, B., Mirmont, M., et al. 2018, A\&A, 611, A87

van Diepen, G., \& Dijkema, T. J. 2018, Astrophysics Source Code Library [record ascl:1804.003]

van Haarlem, M. P., Wise, M. W., Gunst, A. W., et al. 2013, A\&A, 556, A2

van Weeren, R. J., Brunetti, G., Brüggen, M., et al. 2016a, ApJ, 818, 204

van Weeren, R. J., Williams, W. L., Hardcastle, M. J., et al. 2016b, ApJS, 223, 2

van Weeren, R. J., Andrade-Santos, F., Dawson, W. A., et al. 2017, Nat. Astron., 1,0005

van Weeren, R. J., de Gasperin, F., Akamatsu, H., et al. 2019, Space Sci. Rev., 215,16

Venturi, T., Giacintucci, S., Dallacasa, D., et al. 2008, A\&A, 484, 327

Venturi, T., Giacintucci, S., \& Dallacasa, D. 2011, JA\&A, 32, 501

Venturi, T., Giacintucci, S., Dallacasa, D., et al. 2013, A\&A, 551, A24

Wilber, A., Brüggen, M., Bonafede, A., et al. 2018, MNRAS, 473, 3536

Williams, W. L., van Weeren, R. J., Röttgering, H. J. A., et al. 2016, MNRAS, 460, 2385

Wright, E. L. 2006, PASP, 118, 1711 